\title{
Dispersive Shock Wave, Generalized Laguerre Polynomials and Asymptotic Solitons of the Focusing Nonlinear Schrödinger Equation
}

\author{
Vladimir KotLYAROV ${ }^{\dagger}$ and Alexander MinAKOV ${ }^{\ddagger}$ \\ $\dagger$ B.Verkin Institute for Low Temperature Physics and Engineering, \\ 47 Nauky Ave., 61103 Kharkiv, Ukraine \\ ‡Institut de Recherche en Mathématique et Physique (IRMP), Université catholique de Louvain (UCL), \\ Chemin du Cyclotron 2, Louvain-la-Neuve, Belgium
}

May 8, 2019

\begin{abstract}
We consider dispersive shock wave to the focusing nonlinear Schrödinger equation generated by a discontinuous initial condition which is periodic or quasi-periodic on the left semi-axis and zero on the right semi-axis. As an initial function we use a finite-gap potential of the Dirac operator given in an explicit form through hyper-elliptic theta-functions. The paper aim is to study the long-time asymptotics of the solution of this problem in a vicinity of the leading edge, where a train of asymptotic solitons are generated. Such a problem was studied in [29] and [30] using Marchenko's inverse scattering technics. We investigate this problem exceptionally using the Riemann-Hilbert problems technics that allow us to obtain explicit formulas for the asymptotic solitons themselves that in contrast with the cited papers where asymptotic formulas are obtained only for the square of absolute value of solution. Using transformations of the main $\mathrm{RH}$ problems we arrive to a model problem corresponding to the parametrix at the end points of continuous spectrum of the Zakharov-Shabat spectral problem. The parametrix problem is effectively solved in terms of the generalized Laguerre polynomials which are naturally appeared after appropriate scaling of the Riemann-Hilbert problem in a small neighborhoods of the end points of continuous spectrum. Further asymptotic analysis give an explicit formula for solitons at the edge of dispersive wave. Thus, we give the complete description of the train of asymptotic solitons: not only bearing envelope of each asymptotic soliton, but its oscillating structure are found explicitly. Besides the second term of asymptotics describing an interaction between these solitons and oscillating background is also found. This gives the fine structure of the edge of dispersive shock wave.
\end{abstract}

\section{Contents}

1 Introduction

2 Definition of the planar matrix Baker-Akhiezer function and finite-gap solution of the NLS equations.

3 Eigenfunctions

4 The Basic Riemann-Hilbert Problem 


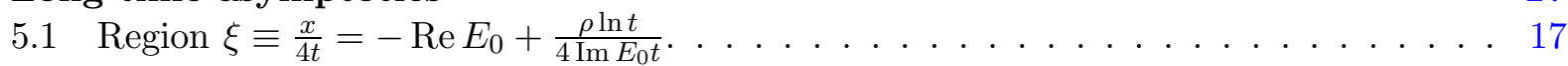

5.1.1 Local changes of variables in the vicinity of the points $k=E_{0}, k=\bar{E}_{0}$. . . . 20

5.1.2 Generalized Laguerre polynomials of index $\frac{1}{2}$. . . . . . . . . . . . . . . . 21

5.1.3 Local change of variable in the vicinity of the point $k=k_{0} \equiv-\xi$. . . . . . . 22

5.1.4 Parabolic cylinder functions. . . . . . . . . . . . . . . . . . 23

5.2 First approximation of $M^{(1)}$. . . . . . . . . . . . . . . . . . . . . . . . . 24

5.2.1 Refined approximation of $M^{(3)}$. . . . . . . . . . . . . . . . . . 26

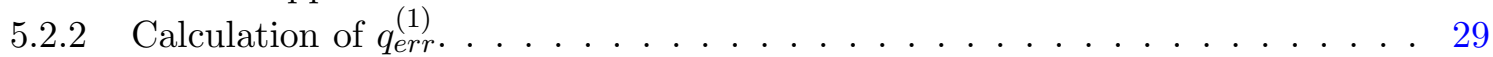

5.2.3 Second refined approximation of $M^{(3)}$. . . . . . . . . . . . . . . . . . 31

\section{Introduction}

Despite many years of an intensive study the range of associated with the nonlinear Schrödinger equation problems continues to expand steadily. This is due to the important theoretical and applied meaning of the nonlinear Schrödinger equation in modern mathematical physics. In recent years there are many interesting problems related to the theory of modulation instability and rogue waves on a deep water, as well as communication systems in nonlinear fibre channels (sf. [5], [6], [20], [21], [23], [35], [36], [38], [27]). In particular, there arise problems of localized perturbations of the periodic or quasi-periodic background. In the present paper we are considering the Cauchy problem for the focusing nonlinear Schrödinger equation with nonlocal perturbations of periodic (quasi-periodic) initial function. Such a problem is well-known in the theory of dispersive shock waves, which have also stable interest of researchers (sf. [3] - [11], [26] - [30], [37]). A good review [23] on this theme represents results of the last 50 years research using the framework of nonlinear modulation theory.

More precisely, we consider the dispersive shock waves of the focusing nonlinear Schrödinger equation. They are generated by a discontinuous initial conditions which are periodic or quasiperiodic on the left semi-axis and zero on the right semi-axis. The paper aim is to study the long-time asymptotics of the solution of this problem in a vicinity of the leading edge, where a train of asymptotic solitons are generated. Such a problem was studied in [29] using Marchenko's inverse scattering techniques under reflectionless condition. This condition was eliminated in [30] where asymptotics was obtained with nonzero reflection coefficient. In this paper we investigate this problem exceptionally using the Riemann-Hilbert problems techniques that allow us to obtain the explicit formulas for asymptotic solitons themselves in contrast with the cited papers where asymptotic formulas are obtained only for the square of absolute value of solution. Thus, we give the complete description of the train of asymptotic solitons: not only bearing envelope of each asymptotic soliton, but its oscillating structure are found explicitly. Besides, the second term of the asymptotics describing an interaction between these solitons and oscillating background is also found. In other words, the paper describes the fine structure of the edge of dispersive shock wave. A sector of $x t$-plane of the leading edge of the wave has essential meaning not only in a study of the structure of dispersive shock waves, but it is very important in itself. It is due to the end-point parametrix problem of the Deift-Zhou method of steepest descent ([12] - [19]). This parametrix problem is effectively solved in terms of the generalized Laguerre polynomials which are naturally appeared after appropriate scaling of the Riemann-Hilbert problem in a small neighborhood of the end points of the continuous spectrum of the Zakharov-Shabat spectral problem. Further asymptotic analysis give a description of a mutual influence between the two parametrices located at the stationary point and the end-point of continuous spectrum. The results may be also interesting for the theory of modulation instability. The first result in this direction for the mKdV equation with constant step-like initial function was obtained in [3] by M.Bertola and one of the author of 
the present paper.

Here we consider a pure step-like initial value problem for the focusing nonlinear Schrödinger equation (however, see a remark at the end of this section):

$$
\begin{gathered}
\mathrm{i} q_{t}+q_{x x}+2|q|^{2} q=0, \quad x \in \mathbb{R}, t \in \mathbb{R}_{+}, \\
q(x, 0)=q_{0}(x)= \begin{cases}0, & x \geq 0 \\
q_{p}(x), & x<0,\end{cases}
\end{gathered}
$$

where $q_{p}(x)$ is a finite-gap periodic or quasi-periodic potential of the Dirac operator (1.3). We will show that the solution of IBV problem (1.1)-(1.2) does exist and unique.

The main tool for studying rigorously the long-time asymptotics of solutions of initial and initial boundary value problems for integrable nonlinear equations is the asymptotic analysis of the Riemann-Hilbert (RH) problem by the Deift-Zhou method of steepest descent [12]. This involves the Jost type solutions of the system of linear equations (the Lax pair or AKNS equations) associated with the nonlinear equation. For the focusing NLS equation (1.1), the Lax pair is as follows [39]:

$$
\begin{gathered}
\Phi_{x}+\mathrm{i} k \sigma_{3} \Phi=Q(x, t) \Phi, \\
\Phi_{t}+2 \mathrm{i} k^{2} \sigma_{3} \Phi=\tilde{Q}(x, t, k) \Phi,
\end{gathered}
$$

where $\sigma_{3}:=\left(\begin{array}{cc}1 & 0 \\ 0 & -1\end{array}\right), \Phi(x, t, k)$ is a $2 \times 2$ matrix-valued function, $k \in \mathbb{C}$ is a spectral parameter, and the matrix coefficients $Q$ and $\tilde{Q}$ are expressed in terms of a scalar function $q$ :

$$
\begin{gathered}
Q(x, t):=\left(\begin{array}{cc}
0 & q(x, t) \\
-\bar{q}(x, t) & 0
\end{array}\right), \\
\tilde{Q}(x, t, k):=2 k Q(x, t)-\mathrm{i}\left(Q^{2}(x, t)+Q_{x}(x, t)\right) \sigma_{3} .
\end{gathered}
$$

It is well-known [1], [24], [39] that this over-determined system of equations is compatible if and only if $q(x, t)$ solves the nonlinear Schrödinger equation (1.1).

The Zakharov-Shabat spectral problem (1.3) with quasi-periodic potential (1.2) $q_{0}(x)$ (of genus $n$ ) possesses an absolutely continuous spectrum $\sigma$ consisting of the real axis $\mathbb{R}$ and a finite number $(n+1)$ of analytical arcs on the complex $k$-plane. The set of eigenvalues is indeed empty for any pure step function $q_{0}(x)$ of zero genus $(n=0)$. For pure step functions of highest genuses $(n \geq 1)$ the number of eigenvalues is not more than $n$, but their set will be empty under special choice of frequencies of the quasi-periodic component of $q_{0}(x)$ (see remark at the end of Section 3). For simplicity we suppose that the set of discrete spectrum is empty. Define a real number $C$ by the relation

$$
C=\max _{k \in \sigma \backslash \mathbb{R}}(-4 \operatorname{Re} k)=-4 \operatorname{Re} E_{0} ; \quad E_{0}=A+\mathrm{i} B \quad B>0 .
$$

Then the main result can be written as follows.

Theorem 1. For $x=C t-\frac{\rho \ln t}{B}$,

- if $\rho \in\left[0, \frac{1}{4}\right)$, then $q(x, t)=q_{p a r}(x, t)+\mathcal{O}\left(t^{-1}\right)$,

- if $\rho \in\left[n+\frac{1}{4}, n+\frac{5}{4}\right), 0 \leq n \in \mathbb{Z}$, then

$$
q(x, t)=q_{s o l}(x, t)+q_{p a r}(x, t)+\mathcal{O}\left(t^{-1} \ln t\right) .
$$

where

$$
q_{s o l}(x, t)=\frac{(-1)^{n} 2 B \exp \left[-2 \mathrm{i}\left(A x+2 t\left(A^{2}-B^{2}\right)\right)-\mathrm{i} \arg \hat{\phi}\left(E_{0}\right)+2 \mathrm{i} \arg \delta\left(E_{0}, A\right)\right]}{\cosh \left[2 B(x+4 A t)+\left(2 n+\frac{3}{2}\right) \ln t+\ln \left(\frac{2 \pi\left|\delta\left(E_{0}, A\right)\right|^{2}}{n ! \Gamma\left(n+\frac{3}{2}\right)} \cdot \frac{\left(16 B^{2}\right)^{2 n+\frac{3}{2}}}{\left|\hat{\phi}\left(E_{0}\right)\right| \sqrt{2 B}}\right)\right]} .
$$


The constant $\hat{\phi}\left(E_{0}\right)$ is determined by the initial datum, and is equal to

$$
\hat{\phi}\left(E_{0}\right)=\lim _{k \rightarrow E_{0}-\mathrm{i} 0} \frac{f(k) \mathrm{e}^{\pi \mathrm{i} / 4}}{\sqrt{\mathrm{i}\left(k-E_{0}\right)}}, \quad \text { where } \quad f(k)=r(k-0)-r(k+0), k \in\left[E_{0}, \operatorname{Re} E_{0}\right] .
$$

where the root is positive for $k \in\left(E_{0}, \operatorname{Re} E_{0}\right)$ and $r(k)$ is the reflection coefficient. Furthermore,

$$
\delta\left(E_{0}, A\right)=\exp \left[\frac{1}{2 \pi \mathrm{i}} \int_{-\infty}^{A} \frac{\ln \left(1+|r(s)|^{2}\right) \mathrm{d} s}{s-A-\mathrm{i} B}\right]
$$

and $\Gamma(n+3 / 2)$ is the Gamma-function. Here $q_{p a r}(x, t)$ is a function that admits the estimate $q_{\text {par }}(x, t)=\mathcal{O}\left(t^{-1 / 2}\right)$. All the estimates are uniform w.r.t. bounded $\rho$.

Theorem 2. The error term in Theorem 1 can be written as

$$
q_{p a r}(x, t)=\sqrt{\frac{\nu}{2 t}}\left(\mathrm{e}^{\mathrm{i} \psi}\left(1-\left|\frac{q_{\text {sol }}(x, t)}{2 B}\right|^{2}\right)-\mathrm{e}^{-\mathrm{i} \psi}\left(\frac{q_{\text {sol }}^{2}(x, t)}{4 B^{2}}\right)\right)+\mathcal{O}\left(\frac{\ln t}{t}\right),
$$

where

$$
\psi:=4 t \xi^{2}+\nu \ln (8 t)+2 \arg \chi\left(k_{0}\right)-\arg r\left(k_{0}\right)-\arg \Gamma(\mathrm{i} \nu)+\frac{\pi}{4},
$$

and

$$
\begin{aligned}
\chi\left(k_{0}, \xi\right) & =\lim _{k \rightarrow k_{0}}\left(k-k_{0}\right)^{\mathrm{i} \nu} \cdot \exp \left[\frac{1}{2 \pi \mathrm{i}} \int_{-\infty}^{k_{0}} \frac{\ln \left(1+|r(s)|^{2}\right) \mathrm{d} s}{s-k}\right] \\
& =(k+N)^{\mathrm{i} \nu} \cdot \exp \left[\frac{1}{2 \pi \mathrm{i}} \int_{-N}^{k_{0}} \frac{\ln \frac{1+|r(s)|^{2}}{1+\left|r\left(k_{0}\right)\right|^{2}} \mathrm{~d} s}{s-k_{0}}+\frac{1}{2 \pi \mathrm{i}} \int_{-\infty}^{-N} \frac{\ln \left(1+|r(s)|^{2}\right) \mathrm{d} s}{s-k}\right]
\end{aligned}
$$

with an arbitrary parameter $-N<k_{0}$, which does not change the value of $\chi\left(k_{0}, \xi\right)$, but which is needed to have convergent integrals in the above representation.

Furthermore, $\nu=\frac{1}{2 \pi} \ln \left[1+\left|r\left(k_{0}\right)\right|^{2}\right], k_{0}=-\xi:=\frac{-x}{4 t}$.

Remark. It is easy to see that the error term can be written as

$$
q_{\text {par }}(x, t)=\sqrt{\frac{\nu}{2 t}} \mathrm{e}^{\mathrm{i} \psi}-\sqrt{\frac{2 \nu}{t}} \frac{\cos \left(\psi-\arg q_{s o l}\right)}{\cosh ^{2} \phi} \mathrm{e}^{\mathrm{i} \arg q_{s o l}}+\mathcal{O}\left(\frac{\ln t}{t}\right),
$$

where

$$
\phi=2 B(x+4 A t)+\left(2 n+\frac{3}{2}\right) \ln t+\ln \left(\frac{2 \pi\left|\delta\left(E_{0}, A\right)\right|^{2}}{n ! \Gamma\left(n+\frac{3}{2}\right)} \cdot \frac{\left(16 B^{2}\right)^{2 n+\frac{3}{2}}}{\left|\hat{\phi}\left(E_{0}\right)\right| \sqrt{2 B}}\right)
$$

and

$$
\arg q_{s o l}=\pi n-2\left(A x+2 t\left(A^{2}-B^{2}\right)\right)-\arg \hat{\phi}\left(E_{0}\right)+2 \arg \delta\left(E_{0}, A\right),
$$

or in another form:

$$
q_{p a r}(x, t)=\sqrt{\frac{\nu}{2 t}}\left(\mathrm{e}^{\mathrm{i} \psi} \tanh ^{2} \phi-\frac{\mathrm{e}^{2 \mathrm{i} \arg q_{s o l}-\mathrm{i} \psi}}{\cosh ^{2} \phi}\right)+\mathcal{O}\left(\frac{\ln t}{t}\right) .
$$

In an elegant form these formulas give a description of the mutual interaction between asymptotic solitons and oscillating background. 
For $C=-4 A>0$ our results show that $q_{0}(x)$ generates a dispersive shock wave whose leading edge is described by a train of asymptotic solitons (1.7) running to the right. The results in [29], [30] were obtained exactly under the restriction $C>0$. The Rieman-Hilbert problem technique gives us the result independently on sign $C$ unlike the Marchenko equations, which are effectively applicable only for the case of $C>0$. But in the case $C<0$ the train of asymptotic solitons runs to the left and thus they describe the rear edge of the dispersive wave. Theorem 2 describes an interaction between asymptotic solitons and oscillating background generated by the parametrices of the end-point of continuous spectrum and the stationary point respectively.

Asymptotic behavior of the dispersive shock wave (DSW) between its leading and trailing edges is much more complicated in compare with studied earlier (sf. [10], [22], [31], [32]) where DSW was described by an elliptic modulated wave [10], [22], [31] or hyperelliptic modulated wave of genus 2 [32]. In our problem the hyperelliptic component of step initial function has genus $n$ and, hence, the spectrum of the Zakharov-Shabat spectral problem can be in general very exotic in its geometry. In turn, a complicated geometry of the spectrum provides a complicated structure of DSW which consists of a finite set of hyperelliptic modulated waves of different genuses. Each of this modulated waves are located in their own sector of the $x t$-plane. Thus, DSW has a very complicated "microstructure", especially in domains between the own sectors of DSW in the $x t$ plane where the different hyperelliptic modulated waves must match each other. A full picture of the DSW "microstructure" is an open problem and a subject of future publications.

To finish the introduction we give the mentioned above remark: the results obtained in the paper are also valid for smooth initial functions, which tend to their asymptotic values sufficiently fast. The proof becomes more complicated in view of the non-analyticity of the reflection coefficient. This difficulty is overcome by the well-known methods of the Deift-Zhou theory [14].

\section{Definition of the planar matrix Baker-Akhiezer function and finite-gap solution of the NLS equations.}

To define $q_{p}(x)$ as a finite-gap potential of the Dirac operator we need to introduce the planar matrix Baker-Akhiezer function associated with the AKNS equations (1.3)-(1.4), using results of [33]. Let $\Sigma_{j}:=\left(E_{j}, \bar{E}_{j}\right), j=0,1,2, \ldots, n$ be a set of vertical open intervals on the complex plane $\mathbb{C}$ which constitute an oriented contour $\Sigma$. All $\Sigma_{j}$ are oriented downwards (Figure 1).

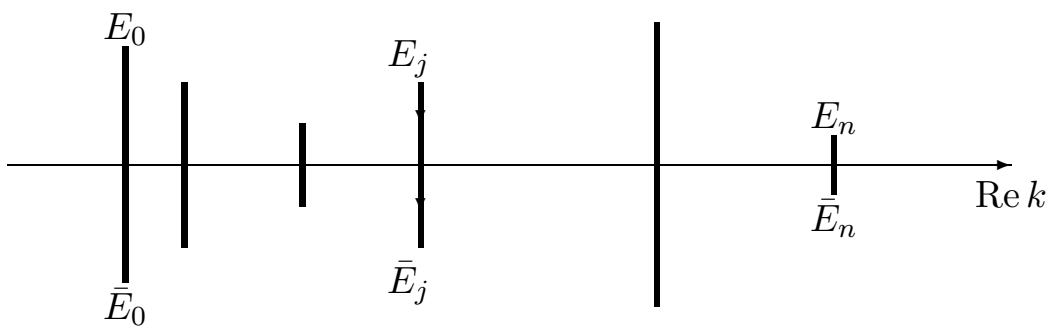

Figure 1: The oriented contour $\Sigma=\cup_{j=1}^{n}\left(E_{j}, \bar{E}_{j}\right)$

Definition 3. Let an oriented contour $\Sigma$ and a set of real numbers $\left(\phi_{1}, \ldots, \phi_{n}\right)$ be given. A $2 \times 2$ matrix $\Phi^{p}(x, t, k)$ is called the Baker-Akhiezer function associated with equations (1.3)-(1.4) if it satisfies following properties:

- for any $x, t \in \mathbb{R}$, the function $\Phi^{p}(x, t, k)$ is analytic in $k \in \mathbb{C} \backslash \tilde{\Sigma}, \quad \tilde{\Sigma}=\cup_{j=0}^{n}\left[E_{j}, \bar{E}_{j}\right]$;

- $\operatorname{det} \Phi^{p}(x, t, k) \equiv 1$;

- $\Phi^{p}(x, t, k)$ has at most the inverse fourth root singularities at $E_{j}$ and $\bar{E}_{j}$; 
- $\Phi^{p}(x, t, k)$ satisfies the jump conditions with piecewise constant jumps:

$$
\Phi_{-}^{p}(x, t, k)=\Phi_{+}^{p}(x, t, k) J_{0}, \quad k \in \Sigma,
$$

where

$$
J_{0}=\left(\begin{array}{cc}
0 & \mathrm{i} e^{-\mathrm{i} \phi_{j}} \\
\mathrm{i} e^{\mathrm{i} \phi_{j}} & 0
\end{array}\right), \quad k \in \Sigma_{j}=\left(E_{j}, \bar{E}_{j}\right), j=0,1, \ldots, n
$$

with $\phi_{0}=0$,

- $\Phi^{p}(x, t, k)=\left(I+O\left(k^{-1}\right)\right) e^{-\mathrm{i}\left(k x+2 k^{2} t\right) \sigma_{3}}$ as $k \rightarrow \infty$.

By the Liouville theorem, the above conditions determine $\Phi_{p}$ uniquely. This function solves the AKNS equations (1.3)-(1.4) with

$$
Q:=Q_{p}(x, t):=\left(\begin{array}{cc}
0 & q_{p}(x) \\
-\bar{q}_{p}(x) & 0
\end{array}\right)
$$

The explicit construction of $\Phi^{p}$ and $q_{p}(x, t)$ is presented in [33]. In particulary, the finite-gap solution $q_{p}(x, t)$ of the nonlinear Schrödinger equation takes the form:

$$
q_{p}(x, t)=2 \mathrm{i} E_{\theta} \frac{\theta\left(-\mathbf{A}(\infty)+\mathbf{A}(\mathcal{D})+\mathbf{K}-\frac{x \mathbf{C}^{h}+t \mathbf{C}^{g}+\phi}{2 \pi}\right)}{\theta\left(\mathbf{A}(\infty)+\mathbf{A}(\mathcal{D})+\mathbf{K}-\frac{x \mathbf{C}^{h}+t \mathbf{C}^{g}+\phi}{2 \pi}\right)} e^{2 \mathrm{i} x h_{0}+2 \mathrm{i} t g_{0}},
$$

where $\theta$ is the well-known theta function, $E_{\theta}$ is a constant:

$$
E_{\theta}:=\frac{1}{2} \sum_{j=0}^{n} \operatorname{Im} E_{j} \frac{\theta(\mathbf{A}(\infty)+\mathbf{A}(\mathcal{D})+\mathbf{K})}{\theta(-\mathbf{A}(\infty)+\mathbf{A}(\mathcal{D})+\mathbf{K})}
$$

and $h_{0}, g_{0}$ are some scalars. Further, $\mathbf{A}(k)$ is the Abel map, $\mathbf{K}$ is the Riemann constant vector. The divisor $\mathcal{D}$ is chosen in such a way that $\Phi_{p}(x, t, k)$ is analytic in $k \in \mathbb{C} \backslash \tilde{\Sigma}$.

The simplest periodic solution is

$$
q_{p}(x, t)=\left(\operatorname{Im} E_{0}\right) \cdot \mathrm{e}^{2 \mathrm{i}\left(x h_{0}+t g_{0}\right)}, \quad h_{0}=-\operatorname{Re} E_{0}, \quad g_{0}=\operatorname{Im}^{2} E_{0}-2 h_{0}^{2} .
$$

The corresponding matrix $\Phi^{p}(x, t, k)$ takes the form [10]:

$$
\Phi^{p}(x, t, k)=\mathrm{e}^{\mathrm{i}\left(x h_{0}+t g_{0}\right) \sigma_{3}} N_{0}(k) \mathrm{e}^{-\mathrm{i}(x h(k)+t g(k)) \sigma_{3}},
$$

where

$$
N_{0}(k)=\frac{1}{2}\left(\begin{array}{ll}
\varkappa_{0}(k)+\frac{1}{\varkappa_{0}(k)} & \varkappa_{0}(k)-\frac{1}{\varkappa_{0}(k)} \\
\varkappa_{0}(k)-\frac{1}{\varkappa_{0}(k)} & \varkappa_{0}(k)+\frac{1}{\varkappa_{0}(k)}
\end{array}\right)
$$

with

$$
\varkappa_{0}(k)=\left(\frac{k-A-\mathrm{i} B}{k-A+\mathrm{i} B}\right)^{\frac{1}{4}}, \quad h(k)=\sqrt{(k-A)^{2}+B^{2}}, \quad g(k)=2(k+A) h(k),
$$

where $A=\operatorname{Re} E_{0}=-h_{0}, B=\operatorname{Im} E_{0}, g_{0}=B^{2}-2 A^{2}$. The branch cut for $\varkappa_{0}$ and $h$ is taken along the vertical segment $[E, \bar{E}]$, where $E=A+\mathrm{i} B$ and $\bar{E}=A-\mathrm{i} B$, and the branches are fixed by asymptotics

$$
h(k)=k-A+\mathrm{O}\left(k^{-1}\right) \quad \text { and } \quad \varkappa_{0}(k)=1+\mathrm{O}\left(k^{-1}\right) \quad \text { as } \quad k \rightarrow \infty .
$$


Notice that $g(k)=2 k^{2}+g_{0}+\mathrm{O}\left(k^{-1}\right)$ with $g_{0}$ given above. In this simplest case the asymptotics were studied in [10], however without analyzing of interjacent sectors.

In general case the Baker-Akhiezer function takes the form [33]:

$$
\Phi^{p}(x, t, k)=\mathrm{e}^{\left(\mathrm{i} h_{0} x+\mathrm{i} g_{0} t\right) \sigma_{3}} N(x, t, k) \mathrm{e}^{-(\mathrm{i} h(k) x+\mathrm{i} g(k) t) \sigma_{3}},
$$

where

$$
h(k)=\frac{w(k)}{2 \pi \mathrm{i}} \sum_{j=1}^{n} \int_{\Sigma_{j}} \frac{C_{j}^{h}}{w_{+}(\xi)(\xi-k)} d \xi, \quad g(k)=\frac{w(k)}{2 \pi \mathrm{i}} \sum_{j=1}^{n} \int_{\Sigma_{j}} \frac{C_{j}^{g}}{w_{+}(\xi)(\xi-k)} d \xi .
$$

The branch of $w(k):=\sqrt{\prod_{j=0}^{n}\left(k-E_{j}\right)\left(k-\bar{E}_{j}\right)}$ is defined as an analytic outside the arcs $\tilde{\Sigma}$ with asymptotics $w(k) \simeq k^{n+1}$ as $k \rightarrow \infty$. Real numbers $C_{j}^{h}$ and $C_{j}^{g}$ are defined in such a way that $h(k)$ and $g(k)$ have the asymptotics:

$$
h(k)=k+h_{0}+O(1 / k), \quad g(k)=2 k^{2}+g_{0}+O(1 / k), \quad k \rightarrow \infty .
$$

Then $C_{j}^{h}$ for $j=1, \ldots, n$ have to satisfy the system of $n$ linear algebraic equations:

$$
\begin{aligned}
& \sum_{j=1}^{n} C_{j}^{h} \int_{\Sigma_{j}} \frac{\xi^{k} \mathrm{~d} \xi}{w_{+}(\xi)}=0, \quad k=0, \ldots, n-2, \\
& \sum_{j=1}^{n} C_{j}^{h} \int_{\Sigma_{j}} \frac{\xi^{n-1} \mathrm{~d} \xi}{w_{+}(\xi)}=-2 \pi \mathrm{i} .
\end{aligned}
$$

This system has a unique solution $\left\{C_{j}^{h}\right\}_{j=1}^{n}$ (sf.[2]). For $C_{j}^{g}$ the system reads

$$
\begin{aligned}
& \sum_{j=1}^{n} C_{j}^{g} \int_{\Sigma_{j}} \frac{\xi^{k} \mathrm{~d} \xi}{w_{+}(\xi)}=0, \quad k=0, \ldots, n-3, \\
& \sum_{j=1}^{n} C_{j}^{g} \int_{\Sigma_{j}} \frac{\xi^{n-2} \mathrm{~d} \xi}{w_{+}(\xi)}=-4 \pi \mathrm{i}, \\
& \sum_{j=1}^{n} C_{j}^{g} \int_{\Sigma_{j}} \frac{\xi^{n-1} \mathrm{~d} \xi}{w_{+}(\xi)}=-2 \pi \mathrm{i} \sum_{j=0}^{n}\left(E_{j}+\hat{E}_{j}\right)
\end{aligned}
$$

These equations have also a unique solution (sf.[2]).

The matrix $N(x, t, k)$ has an explicit representation in theta functions:

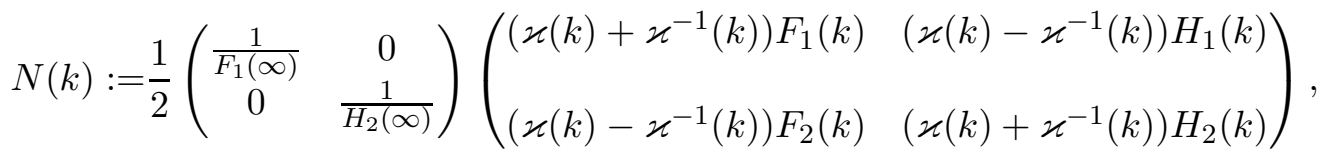

where an analytic in $k \in \mathbb{C} \backslash \tilde{\Sigma}$ function $\varkappa(k)=\prod_{j=0}^{n} \sqrt[4]{\frac{k-E_{j}}{k-\bar{E}_{j}}}$ is defined by cuts $\Sigma_{j}$ and asymptotics $\varkappa(k)=1+\mathrm{O}\left(k^{-1}\right)$ as $k \rightarrow \infty$. For $s=1,2$, the functions $F_{s}(k)$ and $H_{s}(k)$ are as follows:

$$
F_{s}(k)=\frac{\theta\left(\mathbf{A}(k)+\mathbf{C}(\mathbf{x}, \mathbf{t})+\mathbf{d}_{s}\right)}{\theta\left(\mathbf{A}(k)+\mathbf{d}_{s}\right)}, \quad H_{s}(k)=\frac{\theta\left(-\mathbf{A}(k)+\mathbf{C}(\mathbf{x}, \mathbf{t})+\mathbf{d}_{s}\right)}{\theta\left(-\mathbf{A}(k)+\mathbf{d}_{s}\right)} .
$$

with

$$
\mathbf{C}(x, t):=-\frac{x \mathbf{C}^{h}+t \mathbf{C}^{g}+\phi}{2 \pi}, \quad d_{1}=-d_{2}=\mathbf{A}(\mathcal{D})+\mathbf{K}
$$


As usual, the entries of the matrix $N(x, t, k)$ are not independent, namely: $N_{22}(x, t, \bar{k})=\bar{N}_{11}(x, t, k)$ and $N_{21}(x, t, \bar{k})=-\bar{N}_{12}(x, t, k)$. It means that $\operatorname{det} N(x, t, k)=\left|N_{11}(x, t, k)\right|^{2}+\left|N_{12}(x, t, k)\right|^{2} \equiv 1$ for any real $x, t$ and $k \in \mathbb{R}$. Hence, $N_{j l}(x, t, k)(j, l=1,2)$ are uniformly bounded for any real $x$, $t, k$. They are also bounded for any real $x, t$ and $k \in\left[e_{j}, \bar{e}_{j}\right] \subset\left(E_{j}, \bar{E}_{j}\right), j=0,1, \ldots, n$. Since $\operatorname{Im} h(k)=\operatorname{Im} g(k)=0$ for $k \in \Gamma$, where $\Gamma=\mathbb{R} \cup \cup_{j=0}^{n}\left(E_{j}, \bar{E}_{j}\right)$, we have that $\Phi^{\mathrm{p}}(x, t, k)$ is bounded and smooth in $x, t \in \mathbb{R}$ for any fixed $k \in \Gamma$ (with the exception of the points $E_{j}$ and $\bar{E}_{j}$ where entries of $\Phi^{\mathrm{P}}$ have the inverse fourth root singularities).

\section{Eigenfunctions}

Let $q(x, t)$ be a solution to the problem (1.1)-(1.2) satisfying the asymptotic conditions (3.1) and let $Q(x, t)$ and $Q_{\mathrm{p}}(x, t)$ be defined in terms of respectively $q$ and $q_{\mathrm{p}}$ by $(1.5)$.

Assuming for a moment that the function $q(x, t)$ satisfies:

$$
\int_{-\infty}^{0}(1+|x|)\left|q(x, t)-q_{p}(x, t)\right| d x+\int_{0}^{\infty}(1+|x|)|q(x, t)| d x<\infty .
$$

for all $t \geq 0$, we can define the $2 \times 2$-valued functions $\mu_{j}(x, t, k), j=1,2,-\infty<x<\infty, 0 \leq t<\infty$, as the solutions of the Volterra integral equations:

$$
\begin{aligned}
& \mu_{1}(x, t, k)=I-\int_{x}^{\infty} \mathrm{e}^{\mathrm{i} k(y-x) \sigma_{3}}\left(Q \mu_{1}\right)(y, t, k) \mathrm{e}^{-\mathrm{i} k(y-x) \sigma_{3}} \mathrm{~d} y, \quad k \in \mathbb{R}, \\
& \mu_{2}(x, t, k)=I+\int_{-\infty}^{x} G^{\mathrm{p}}(x, y, t, k)\left[Q(y, t)-Q_{\mathrm{p}}(y, t)\right] \mu_{2}(y, t, k)\left[G^{\mathrm{p}}(x, y, t, k)\right]^{-1} \mathrm{~d} y, \quad k \in \Gamma,
\end{aligned}
$$

where $G^{\mathrm{p}}(x, y, t, k)$ is given by

$$
G^{\mathrm{p}}(x, y, t, k)=\Phi^{\mathrm{P}}(x, t, k)\left[\Phi^{\mathrm{P}}(y, t, k)\right]^{-1} .
$$

Obviously, for real $x$ and $t, G^{\mathrm{p}}(x, y, k)$ is an analytic function in $k \in \mathbb{C} \backslash \tilde{\Gamma}$, where $\tilde{\Gamma}$ is a closure of $\Gamma$, i.e.: $\tilde{\Gamma}:=\mathbb{R} \cup \cup_{j=0}^{n}\left[E_{j}, \bar{E}_{j}\right]$. It has the asymptotic behavior for a large $k$ :

$$
G^{\mathrm{p}}(x, y, k)=\mathrm{e}^{\mathrm{i}(y-x) h(k) \sigma_{3}}\left[I+\mathrm{O}\left(\frac{1}{k}\right)\right] \quad \text { as } k \rightarrow \infty, \quad \operatorname{Im} h(k)=0 .
$$

The analytic properties of $\mu_{j}$ are collected in the following

Proposition. The $2 \times 2$ matrices $\mu_{j}(x, t, k), j=1,2$ have the following properties:

(i) $\operatorname{det} \mu_{1}(x, t, k)=\operatorname{det} \mu_{2}(x, t, k) \equiv 1$.

(ii) The functions $\Psi(x, t, k)$ and $\Phi(x, t, k)$ defined by

$$
\begin{aligned}
& \Psi(x, t, k):=\mu_{1}(x, t, k) \mathrm{e}^{-\mathrm{i} k x \sigma_{3}-2 \mathrm{i} k^{2} t \sigma_{3}}, \\
& \Phi(x, t, k):=\mu_{2}(x, t, k) \mathrm{e}^{-\mathrm{i} x h(k) \sigma_{3}-\mathrm{i} t g(k) \sigma_{3}}
\end{aligned}
$$

satisfy the Lax pair equations (1.3)-(1.4).

(iii) Let the columns of $a 2 \times 2$ matrix $M$ be denoted respectively by $M^{(1)}$ and $M^{(2)}$. Then $\mu_{1}^{(1)}(x, t, k)$ is analytic in $k \in \mathbb{C}_{-}$and $\mu_{1}^{(1)}(x, t, k)=\left(\begin{array}{l}1 \\ 0\end{array}\right)+\mathrm{O}\left(k^{-1}\right)$ as $k \rightarrow \infty, \operatorname{Im} k \leq 0$ whereas $\mu_{1}^{(2)}(x, t, k)$ is analytic in $k \in \mathbb{C}_{+}$and $\mu_{1}^{(2)}(x, t, k)=\left(\begin{array}{l}0 \\ 1\end{array}\right)+\mathrm{O}\left(k^{-1}\right)$ as $k \rightarrow \infty, \operatorname{Im} k \geq 0$. 
(iv) $\mu_{2}^{(1)}(x, t, k)$ is analytic in $k \in \mathbb{C}_{+} \backslash \tilde{\Sigma}_{+}$, has a jump across $\tilde{\Sigma}_{+}:=\tilde{\Sigma} \cap \mathbb{C}_{+}$, and $\mu_{2}^{(1)}(x, t, k)=$ $\left(\begin{array}{l}1 \\ 0\end{array}\right)+\mathrm{O}\left(k^{-1}\right)$ as $k \rightarrow \infty, \operatorname{Im} k \geq 0$ whereas $\mu_{2}^{(2)}(x, t, k)$ is analytic in $k \in \mathbb{C}_{-} \backslash \tilde{\Sigma}_{-}$, has a jump across $\tilde{\Sigma}_{-}:=\tilde{\Sigma} \cap \mathbb{C}_{-}$, and $\mu_{2}^{(2)}(x, t, k)=\left(\begin{array}{l}0 \\ 1\end{array}\right)+\mathrm{O}\left(k^{-1}\right)$ as $k \rightarrow \infty, \operatorname{Im} k \leq 0$.

(v) Moreover,

$$
\mu_{j}(x, t, k)=I+\frac{\tilde{\mu}(x, t)}{\mathrm{i} k}+\mathrm{o}\left(k^{-1}\right)
$$

as $k \rightarrow \infty$ along curves non-tangential to $\mathbb{R}$ (the expansion is to be understood column-wise, in the respective half-plane of the $k$-plane), where

$$
\left[\sigma_{3}, \tilde{\mu}(x, t)\right]=\left(\begin{array}{cc}
0 & q(x, t) \\
-\bar{q}(x, t) & 0
\end{array}\right) .
$$

(vi) Near $k=E_{j}$ and $k=\bar{E}_{j}$, the respective columns of $\mu_{2}(x, t, k)$ exhibit inverse fourth-root singularities like those the matrix $M(x, t, k)$ has.

Since the eigenfunctions $\Psi(x, t, k)$ and $\Phi(x, t, k)$ satisfy both equations of the Lax pair, we have

$$
\Phi(x, t, k)=\Psi(x, t, k) S(k), \quad k \in \mathbb{R},
$$

where $S(k)$ is independent of $x$ and $t$. In the absence of any perturbations of the discontinuous initial function we have

$$
\Psi(x, 0, k)=\mathrm{e}^{-\mathrm{i} k x \sigma_{3}}
$$

for $x \geq 0$ and

$$
\Phi(x, 0, k)=\Phi^{\mathrm{p}}(x, 0, k)
$$

for $x \leq 0$. Therefore

$$
S(k):=\left(\begin{array}{cc}
a(k) & -\bar{b}(k) \\
b(k) & \bar{a}(k)
\end{array}\right)=\Psi^{-1}(0,0, k) \Phi^{\mathrm{P}}(0,0, k)=\Phi^{\mathrm{P}}(0,0, k)=N(0,0, k) .
$$

where

$$
\begin{aligned}
& a(k)=N_{11}(0,0, k)=\bar{N}_{22}(0,0, \bar{k})=\frac{\varkappa(k)+\varkappa^{-1}(k)}{2} \frac{F_{1}^{0}(k)}{F_{1}^{0}(\infty)}, \\
& b(k)=N_{21}(0,0, k)=-\bar{N}_{12}(0,0, \bar{k})=\frac{\varkappa(k)-\varkappa^{-1}(k)}{2} \frac{F_{2}^{0}(k)}{H_{2}^{0}(\infty)},
\end{aligned}
$$

and

$$
F_{s}^{0}(k)=\frac{\theta\left(\mathbf{A}(k)+\mathbf{C}(\mathbf{0}, \mathbf{0})+\mathbf{d}_{s}\right)}{\theta\left(\mathbf{A}(k)+\mathbf{d}_{s}\right)}, \quad H_{s}^{0}(k)=\frac{\theta\left(-\mathbf{A}(k)+\mathbf{C}(\mathbf{0}, \mathbf{0})+\mathbf{d}_{s}\right)}{\theta\left(-\mathbf{A}(k)+\mathbf{d}_{s}\right)}, \quad s=1,2
$$

with

$$
\mathbf{C}(0,0):=-\frac{\phi}{2 \pi}, \quad d_{1}=-d_{2}=\mathbf{A}(\mathcal{D})+\mathbf{K} .
$$

Thus, for the discontinuous initial function, $a(k)$ and $b(k)$ are analytic in $k \in \mathbb{C} \backslash \tilde{\Sigma}, \quad \tilde{\Sigma}=$ $\cup_{j=0}^{n}\left[E_{j}, \bar{E}_{j}\right]$ and possess following symmetries:

$$
\bar{a}(\bar{k})=a(k), \quad \bar{b}(\bar{k})=-b(k) .
$$

The behavior at infinity are:

$$
a(k)=1+\mathrm{O}\left(\frac{1}{k}\right) \quad \text { as } k \rightarrow \infty, \quad b(k)=\mathrm{O}\left(\frac{1}{k}\right) \quad \text { as } k \rightarrow \infty .
$$


The functions $F_{1}^{0}(k)$ and $F_{2}^{0}(k)$ are analytic in $k \in \mathbb{C} \backslash \tilde{\Sigma}$, continuous and bounded up to the contour $\tilde{\Sigma}$. Hence $a(k)$ and $b(k)$ are also analytic in $k \in \mathbb{C} \backslash \tilde{\Sigma}$ and continuous up to $\Sigma$ with the exception of the end points $E_{j}$ and $\bar{E}_{j}$ where they have forth root singularities. Their ratio $r(k):=b(k) / a(k)$, i.e. the reflection coefficient is continuous and bounded on the both sides of contour $\tilde{\Sigma}$ (the singularities are compensated). In general, $a(k)$ may have zeros at the points $\left\{k_{1}, k_{2}, \ldots, k_{m}\right\}(m \leq n)$ which coincide with projections of zeros of $\theta(\mathbf{A}(k)+\mathbf{C}(\mathbf{0}, \mathbf{0})+\mathbf{A}(\mathcal{D})+\mathbf{K})$. This function has precisely $n$ zeros which lies on the Riemann surface ( $m$ of them on the upper sheet, and $n-m$ on the lower sheet). On the other hand, one can control these zeros. For example, let $\mathcal{D}_{\phi}$ be a non-special divisor on the upper sheet. Let us choose free parameters $\left(\phi_{1}, \ldots, \phi_{n}\right)$ of the initial function $q_{p}(x)$ in such a way that $a(k) \neq 0$. It will be done if $\mathcal{D}_{\phi} \cap \mathcal{D}=\emptyset$ and $\phi_{j}=2 \pi\left(\mathbf{A}_{j}(\mathcal{D})-\mathbf{A}_{j}\left(\mathcal{D}_{\phi}\right)\right), j=1,2, \ldots, n$ because all zeros of the corresponding theta-function will be situated on the lower sheet. Thus, $a(k)$ may have zeros, but not more than the corresponding genus $(n)$ of the Riemann surface even in the case of discontinuous pure step initial function $q(x, 0)$. In what follows we consider the case $a(k) \neq 0$.

\section{The Basic Riemann-Hilbert Problem}

The scattering relation (3.3) involving the eigenfunctions $\Psi(x, t, k)$ and $\Phi(x, t, k)$ can be rewritten in the form of conjugation of boundary values of a piecewise analytic matrix-valued function on a contour in the complex $k$-plane, namely:

$$
M_{-}(x, t, k)=M_{+}(x, t, k) J(x, t, k), \quad k \in \Gamma=\mathbb{R} \cup \cup_{j=0}^{n}\left(E_{j}, \bar{E}_{j}\right),
$$

where $M_{ \pm}(x, t, k)$ denote the boundary values of $M(x, t, k)$ according to a chosen orientation of $\Gamma$, i.e. $M_{ \pm}(x, t, k)$ is a non-tangential limit of $M\left(x, t, k^{\prime}\right)$ as $k^{\prime} \rightarrow k \in \Gamma$, from the positive/negative side of the contour $\Gamma$ (see Figure 2).

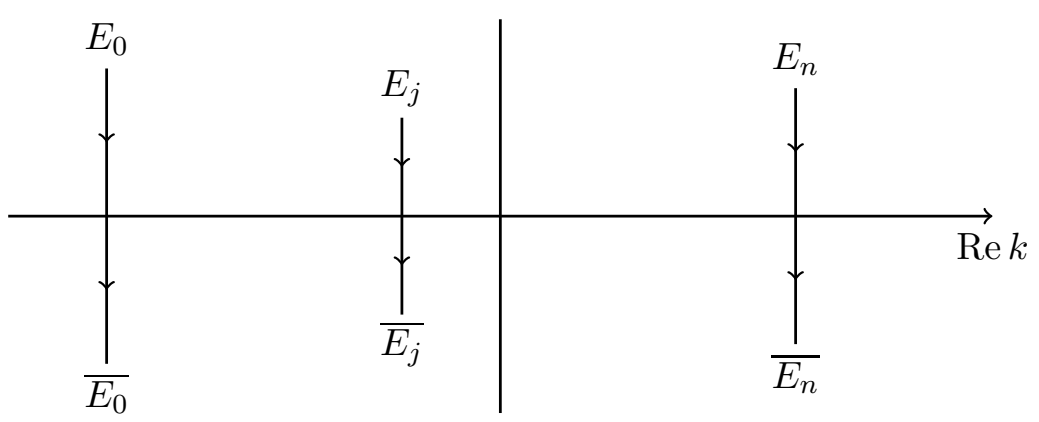

Figure 2: The oriented contour $\Gamma=\mathbb{R} \cup \Sigma$

Indeed, let us write (3.3) in the vector form:

$$
\begin{aligned}
& \frac{\Phi^{(1)}(x, t, k)}{a(k)}=\Psi^{(1)}(x, t, k)+r(k) \Psi^{(2)}(x, t, k), \\
& \frac{\Phi^{(2)}(x, t, k)}{\bar{a}(k)}=-\bar{r}(k) \Psi^{(1)}(x, t, k)+\Psi^{(2)}(x, t, k),
\end{aligned}
$$

where

$$
r(k):=\frac{b(k)}{a(k)}, \quad \bar{r}(k):=\frac{\bar{b}(k)}{\bar{a}(k)}=-r(k),
$$


and define the matrix $M(x, t, k)$ as follows:

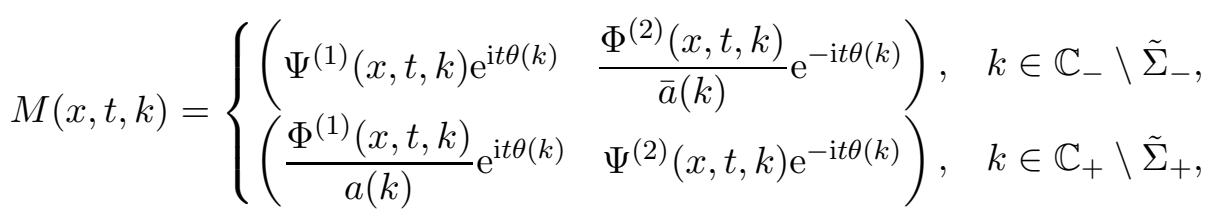

where

$$
\theta(k)=\theta(k ; \xi)=2 k^{2}+4 \xi k \quad \text { with } \xi=\frac{x}{4 t} .
$$

Then the boundary values $M_{-}(x, t, k)$ and $M_{+}(x, t, k)$ are related by $(4.1)$, where

$$
J(x, t, k)=\left\{\begin{array}{l}
\left(\begin{array}{cc}
1 & -\bar{r}(k) \mathrm{e}^{-2 \mathrm{i} t \theta(k)} \\
-r(k) \mathrm{e}^{2 \mathrm{i} t \theta(k)} & 1+|r(k)|^{2}
\end{array}\right), \quad k \in \mathbb{R} \backslash \cup_{j=0}^{n}\left\{\operatorname{Re} E_{j}\right\}, \\
\left.\begin{array}{rr}
1 & -\bar{f}(\bar{k}) \mathrm{e}^{-2 \mathrm{i} t \theta(k)} \\
0 & 1
\end{array}\right), \quad k \in \Sigma_{-}, \\
\left(\begin{array}{cc}
1 & 0 \\
f(k) \mathrm{e}^{2 \mathrm{i} t \theta(k)} & 1
\end{array}\right), \quad k \in \bar{\Sigma}_{+},
\end{array}\right.
$$

with

$$
f(k):=r_{-}(k)-r_{+}(k), \quad \bar{f}(\bar{k})=-f(k) .
$$

Jump relation (4.1) considered together with the properties of the eigenfunctions listed in Proposition 3 suggests the way of representing the solution to problem (1.1)-(1.2) in terms of a solution of the following Riemann-Hilbert problem (specified by initial conditions (1.2) via the associated spectral function $r(k))$.

Basic RH problem. Given analytic outside $\tilde{\Gamma}=\mathbb{R} \cup \tilde{\Sigma}$ functions $r(k)=-\bar{r}(\bar{k})$ and $f(k)=$ $r_{-}(k)-r_{+}(k)=-\bar{f}(\bar{k})$ for $k \in \Gamma$, find a $2 \times 2$-valued function $M(x, t, k)$ such that

(i) $M(x, t, k)$ is analytic in $k \in \mathbb{C} \backslash \tilde{\Gamma}$.

(ii) $M(x, t, k)$ has at most the inverse fourth root singularities at $E_{j}$ and $\bar{E}_{j}$;

(iii) the boundary values $M_{ \pm}(x, t, k)$ satisfy the jump condition

$$
M_{-}(x, t, k)=M_{+}(x, t, k) J(x, t, k), \quad k \in \Gamma \backslash\left\{\cup_{j=1}^{n} \operatorname{Re} E_{j}\right\},
$$

where the jump matrix $J(x, t, k)$ is defined in terms of $r(k)$ and $f(k)$ by (4.5);

(iv)

$$
M(x, t, k)=I+\mathrm{O}\left(\frac{1}{k}\right) \quad \text { as } k \rightarrow \infty .
$$

Then the solution $q(x, t)$ of problem (1.1)-(1.2) can be expressed in terms of the solution of the $\mathrm{RH}$ problem (i)-(iv) as follows:

$$
q(x, t)=2 \mathrm{i} \lim _{k \rightarrow \infty}(k M(x, t, k))_{12} .
$$

The basic RH problem has universal structure in the sense that for any initial function $q_{0}(x)$ with prescribed behavior at infinity, and such that the direct scattering problem is well-posed, it has the same form as in Definition with following differences of properties of the scattering data: 
- the discrete spectrum is not empty and residual conditions are presented;

- $f(k)$ and $\bar{f}(\bar{k})$ are some functions given on semi-intervals $\left(\operatorname{Re} E_{j}, E_{j}\right)$ and $\left(\operatorname{Re} E_{j}, \bar{E}_{j}\right)$;

- $r(k)$ is a function given for real $k$ with jumps at the points $E_{j}$ :

$$
r_{-}\left(\operatorname{Re} E_{j}\right)-r_{+}\left(\operatorname{Re} E_{j}\right)=f\left(\operatorname{Re} E_{j}\right), \quad j=0,1,2, \ldots, n ;
$$

At this point we can forget how the Riemann-Hilbert problem (i) -(iv) was deduced. We simply prove that such a problem has a unique solution which is smooth in $x$ and $t \neq 0$. Moreover, we show that the matrix $M(x, t, k)$ generates a solution of the AKNS equations and, as a result, a smooth solution of the focusing nonlinear Schrödinger equation. Just this solution is the subject of our research. In the present paper we restrict our attention to the leading edge of the dispersive shock wave only.

Theorem 4. For any fixed $x, t \in \mathbb{R}$, the Riemann - Hilbert problem (i)-(iv) has the unique solution. This solution is continuous in the parameters $(x, t) \in \mathbb{R} \times \mathbb{R}$.

Proof. Existence. Let $x$ and $t$ be fixed. We look for the solution $M(x, t, k)$ of the RH problem in the form:

$$
M(x, t, k)=I+\frac{1}{2 \pi \mathrm{i}} \int_{\Gamma} \frac{[I+N(x, t, s)][I-J(x, t, s)]}{s-k} \mathrm{~d} s, \quad k \in \mathbb{C} \backslash \Gamma .
$$

One can show that the Cauchy integral (4.8) provides all properties of the RH problem if and only if the matrix $N(x, t, k)$ satisfies the singular integral equation

$$
N(x, t, s)-\mathcal{K}[N](x, t, s)=F(x, t, s) .
$$

The singular integral operator $\mathcal{K}$ and the right-hand side $F(x, t, s)$ are as follows:

$$
\begin{gathered}
\mathcal{K}[N](x, t, s)=\frac{1}{2 \pi \mathrm{i}} \int_{\Gamma} \frac{N(x, t, z)[I-J(x, t, z)]}{z-s_{+}} \mathrm{d} z, \\
F(x, t, s)=\frac{1}{2 \pi \mathrm{i}} \int_{\Gamma} \frac{I-J(x, t, z)}{z-s_{+}} \mathrm{d} z .
\end{gathered}
$$

We consider this integral equation in the space $L^{2}(\Gamma)$ of $2 \times 2$ matrix complex-valued functions $N(k):=N(x, t, s)$. The operator $\mathcal{K}$ is defined by the jump matrix $J(x, t, k)$ and the generalized function $\frac{1}{z-s_{+}}=\lim _{k \rightarrow s, k \in+\text { side }} \frac{1}{z-k}$.

The Cauchy operator

$$
C_{+}[f](s)=\frac{1}{2 \pi \mathrm{i}} \int_{\Gamma} \frac{f(z)}{z-s_{+}} \mathrm{d} z
$$

is bounded in the space $L_{2}(\Gamma)[34]$.

The matrix-valued function $I-J(x, t, k)$ as a function of the variable $k$ is in the space $L_{2}(\Gamma)$. Hence, the function $F(x, t, k)$ is also in $L_{2}(\Gamma)$. The matrix-valued function $I-J(x, t, k)$ is bounded as a function of the variable $k: I-J(x, t, k) \in L_{\infty}(\Gamma)$. Thus $I d-\mathcal{K}$ is a function acting in $L_{2}(\Gamma)$ ( $I d$ is the identical operator). The contour $\Gamma$ and the jump matrix $J(x, t, k)$ satisfy the Schwartz reflection principle [40]:

- the contour $\Gamma$ is symmetric with respect to the real axis $\mathbb{R}$,

- $J(x, t, k)^{-1}=\overline{J(x, t, \bar{k}}^{T}$ for $k \in \Sigma_{+} \cup \Sigma_{-}$, 
- the jump matrix $J(x, t, k)$ has a positive definite real part for $k \in \mathbb{R} \backslash \cup_{j=0}^{n}\left\{\operatorname{Re} E_{j}\right\}$.

Then Theorem 9.3 from [40] (p. 984) guarantees the $L^{2}$ invertibility of the operator $I d-\mathcal{K}$. Therefore, the singular integral equation (4.9) has a unique solution $N(x, t, k) \in L_{2}(\Gamma)$ for any fixed $x, t \in \mathbb{R}$ and the formula (4.8) gives the solution of the above $\mathrm{RH}$ problem.

The operator $I d-\mathcal{K}$ depends continuously on the parameters $(x, t) \in \mathbb{R} \times \mathbb{R}$. Therefore the inverse operator $(I d-\mathcal{K})^{-1}$ also has this property. Hence, the solution $N(x, t, k)$ of singular integral equation (4.9) also depends continuously on $x, t$. From representation(4.8) we obtain the required statement for $M(x, t, k)$.

Uniqueness. The uniqueness for the Riemann - Hilbert problem (i)-(iv) in the space $L_{2}(\Gamma)$ is proved in [19] (p. 194-198).

Theorem 5. For any $x \in \mathbb{R}$ and $t \neq 0$, the solution of the $R H$ problem (i)-(iv) is infinitely differentiable in $x$ and $t$.

Proof. First of all we note that it is impossible to differentiate the equation (4.9) with respect to $x$ and $t$ because the function $r(k)$, as well as the matrix $I-J(x, t, k)$, vanishes as $k^{-1}$ when $k \rightarrow \pm \infty$ along the real $k$-axis. To avoid a weak decreasing of the matrix $I-J(x, t, k)$ for large real $k$, we use an equivalent $\mathrm{RH}$ problem on such a contour, where the jump matrix $I-J(x, t, k)$ for large complex $k$ becomes exponentially small.

We restrict ourselves to the case $t>0$ and $x \in \mathbb{R}$. We pick up two arbitrary real numbers such that $k_{1}<\operatorname{Re} E_{0}$ and $k_{2}>\operatorname{Re} E_{n}$. Let us perform the next transformations. The first one:

$$
M^{(1)}(x, t, k)=M(x, t, k) \delta^{-\sigma_{3}}\left(k, k_{1}\right), \quad \delta\left(k, k_{1}\right)=\exp \left(\frac{1}{2 \pi i} \int_{-\infty}^{k_{1}} \frac{\ln \left(1+|r(s)|^{2}\right) d s}{s-k}\right) .
$$

Then $M_{-}^{(1)}(x, t, k)=M_{+}^{(1)}(x, t, k) J^{(1)}(x, t, k)$ where $J^{(1)}(x, t, k)=\delta^{\sigma_{3}}(k, \xi) J(x, t, k) \delta^{-\sigma_{3}}\left(k, k_{1}\right)$ for $k \in \Sigma_{+} \cup \Sigma_{-}$, and on the real axis

$$
\begin{aligned}
J^{(1)}(x, t, k) & =\left(\begin{array}{ccc}
1 & \frac{r(k)}{1-r^{2}(k)} \delta_{+}^{2}\left(k, k_{1}\right) \mathrm{e}^{-2 \mathrm{i} t \theta(k, \xi)} \\
0 & 1 &
\end{array}\right)\left(\begin{array}{cc}
1 & 0 \\
\frac{-r(k)}{1-r^{2}(k)} \delta_{-}^{-2}\left(k, k_{1}\right) \mathrm{e}^{2 \mathrm{i} t \theta(k, \xi)} & 1
\end{array}\right), \quad k<k_{1} \\
& =\left(\begin{array}{cc}
1 & 0 \\
-r(k) \delta^{-2}\left(k, k_{1}\right) \mathrm{e}^{2 \mathrm{i} t \theta(k, \xi)} & 1
\end{array}\right)\left(\begin{array}{cc}
\begin{array}{cc}
r(k) \delta^{2}\left(k, k_{1}\right) \mathrm{e}^{-2 \mathrm{i} t \theta(k, \xi)} \\
0
\end{array} & 1
\end{array}\right), \quad k>k_{2} .
\end{aligned}
$$

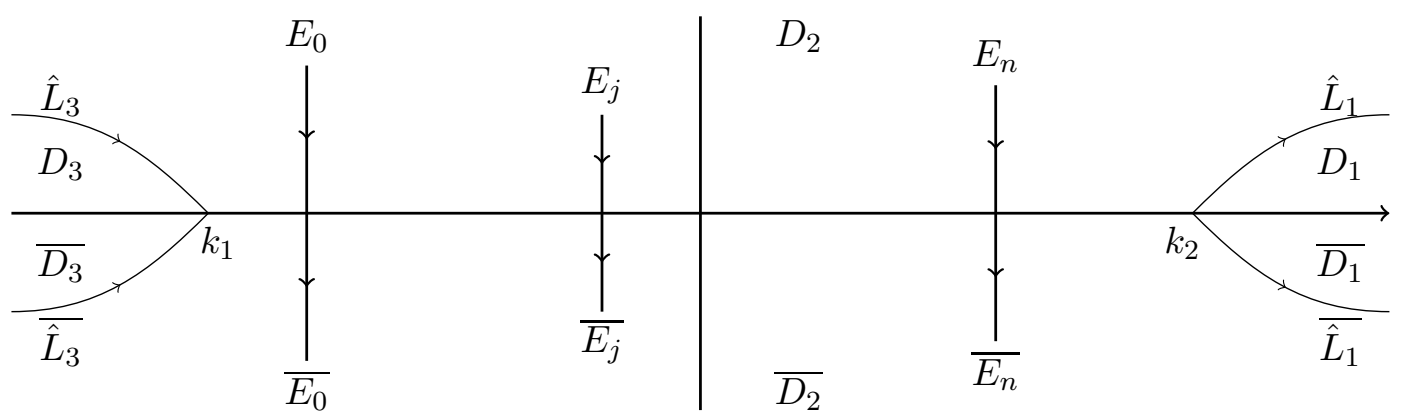

Figure 3: Decomposition of $\mathbb{C}$ into $D_{j}$ and $\overline{D_{j}}, j=1,2,3$.

Define a decomposition (see Fig. 3) of the complex $k$-plane into the six domains $D_{1}, D_{2}, D_{3}$ and their complex conjugated domains $\overline{D_{1}}, \overline{D_{2}}, \overline{D_{3}}$. These domains are separated by the contour 
$\hat{\Sigma}^{(1)}=\mathbb{R} \cup \hat{L}_{1}\left(k_{2}\right) \cup \overline{\hat{L}_{1}\left(k_{2}\right)} \cup \hat{L}_{3}\left(k_{1}\right) \cup \overline{\hat{L}_{3}\left(k_{1}\right)}$, where $\hat{L}_{1}\left(k_{2}\right)=\left\{k: \arg \left(\mathrm{k}-\mathrm{k}_{2}\right)=\pi / 4\right\}$,

$\overline{\hat{L}_{1}\left(k_{2}\right)}=\left\{k: \arg \left(\mathrm{k}-\mathrm{k}_{2}\right)=-\pi / 4\right\}, \hat{L}_{3}\left(k_{1}\right)=\left\{k: \arg \left(\mathrm{k}-\mathrm{k}_{1}\right)=3 \pi / 4\right\}$,

$\overline{\hat{L}_{3}\left(k_{1}\right)}=\left\{k: \arg \left(\mathrm{k}-\mathrm{k}_{1}\right)=-3 \pi / 4\right\}$. The jump matrix $J^{(1)}(x, t, k)$ initiates the second transformation:

$$
M^{(2)}(x, t, k)=M^{(1)}(x, t, k) G^{(1)}(x, t, k),
$$

where

$$
\begin{array}{rlr}
G^{(1)}(x, t, k) & =\left(\begin{array}{cc}
1 & 0 \\
-r(k) \delta^{-2}(k, \xi) \mathrm{e}^{2 \mathrm{i} t \theta(k, \xi)} & 1
\end{array}\right), & k \in D_{1}, \\
& =\left(\begin{array}{cc}
1 & r(k) \delta^{2}(k, \xi) \mathrm{e}^{-\mathrm{i} t \theta(k, \xi)} \\
0 & 1
\end{array}\right), & k \in \overline{D_{1}} \\
& =\left(\begin{array}{ll}
1 & 0 \\
0 & 1
\end{array}\right), & k \in D_{2} \cup \overline{D_{2}}, \\
G^{(1)}(x, t, k) & =\left(\begin{array}{ccc}
1 & \frac{r(k)}{1-r^{2}(k)} \delta_{+}^{2}(k, \xi) \mathrm{e}^{-2 \mathrm{i} t \theta(k, \xi)} \\
0 & 1 & 1
\end{array}\right), \\
& =\left(\begin{array}{cc}
\frac{-r(k)}{1-r^{2}(k)} \delta_{-}^{-2}(k, \xi) \mathrm{e}^{2 \mathrm{i} t \theta(k, \xi)} & 1
\end{array}\right), & k \in D_{3} \\
\end{array}
$$

The $G^{(1)}$-transformation implies the following $\mathrm{RH}$ problem:

$$
\begin{aligned}
M_{-}^{(2)}(x, t, k) & =M_{+}^{(2)}(x, t, k) J^{(2)}(x, t, k), \quad k \in \hat{\Sigma}^{(2)}, \\
& M^{(2)}(x, t, k) \rightarrow I, k \rightarrow \infty
\end{aligned}
$$

where the jump matrix $J^{(2)}(x, t, k)=\left(G^{(1)}(x, t, k)\right)^{-1} J^{(1)}(x, t, k) G^{(1)}(x, t, k)$ for $k \in \Sigma_{+} \cup \Sigma_{-}$and

$$
\begin{aligned}
& J^{(2)}(x, t, k)=\left(\begin{array}{cc}
1 & \frac{r(k)}{1-r^{2}(k)} \delta_{+}^{2}(k, \xi) \mathrm{e}^{-2 \mathrm{i} t \theta(k, \xi)} \\
0 & 1
\end{array}\right), \quad k \in L_{3}\left(k_{1}\right) \\
& =\left(\begin{array}{cc}
-r(k) & 1 \\
\frac{1-r^{2}(k)}{-2}(k, \xi) \mathrm{e}^{2 \mathrm{i} t \theta(k, \xi)} & 1
\end{array}\right), \quad k \in \overline{L_{3}\left(k_{1}\right)}, \\
& =\left(\begin{array}{cc}
1 & 0 \\
-r(k) \mathrm{e}^{2 \mathrm{i} t \theta(k, \xi)} & 1
\end{array}\right), \quad k \in L_{1}\left(k_{2}\right), \\
& =\left(\begin{array}{cc}
1 & -\overline{r(\bar{k})} \mathrm{e}^{-2 \mathrm{i} t \theta(k, \xi)} \\
0 & 1
\end{array}\right), \quad k \in \overline{L_{1}\left(k_{2}\right)}, \\
& =\left(\begin{array}{ll}
1 & 0 \\
0 & 1
\end{array}\right), \quad k \in \mathbb{R} \backslash \bigcup_{j=0}^{n}\left\{\operatorname{Re} E_{j}\right\} .
\end{aligned}
$$

As we did for the matrix $M(x, t, k)$, let us pass to $M^{(2)}(x, t, s)=C\left[I+N^{(2)}\right](x, t, s)$ and to the equivalent singular integral equation:

$$
N^{(2)}(x, t, s)-\frac{1}{2 \pi \mathrm{i}} \int_{\Gamma^{(2)}} \frac{N^{(2)}(x, t, z)\left[I-J^{(2)}(x, t, z)\right]}{z-s_{+}} \mathrm{d} z=\frac{1}{2 \pi \mathrm{i}} \int_{\Gamma^{(2)}} \frac{\left[I-J^{(2)}(x, t, z)\right]}{z-s_{+}} \mathrm{d} z
$$


where $\Gamma^{(2)}=\Sigma_{+} \cup \Sigma_{-} \cup L_{1}(\xi) \cup \overline{L_{1}(\xi)} \cup L_{3}(\xi) \cup \overline{L_{3}(\xi)}$. Like the singular integral equation (4.9), this equation has a unique solution $N^{(2)}(x, t, s) \in L_{2}\left(\Gamma^{(2)}\right)$. Now we can differentiate the last equation in $x$ and $t$ as many times as desired. Indeed, to differentiate these equations and matrix $N^{(2)}(x, t, s)$ it is sufficient that its formal derivatives are convergent. The function $I-J(x, t, s)$ is responsible for decaying of integrands in the first case. In the second case decaying of integrands is determined by $I-J^{(2)}(x, t, s)$. While on the real axis the function $I-J(x, t, s)$ decreases as $1 / s$, which does not allow to differentiate, the function $I-J^{(2)}(x, t, s)$ decreases exponentially on the infinite parts of the contour $\Gamma^{(2)}$ (we remind that $t>0$ ). It provides a unique solvability and existence of the partial derivatives of $N^{(2)}(x, t, k)$ with respect to $x$ and $t$. Hence, the same is true for $M^{(2)}(x, t, k)$ and $M(x, t, k)$.

Theorem 6. Let $\Phi^{(2)}(x, t, k) \equiv M^{(2)}(x, t, k) \mathrm{e}^{\left(\mathrm{i} k x+2 \mathrm{i} k^{2} t\right) \sigma_{3}}$. Then $\Phi^{(2)}(x, t, k)$ satisfies the AblowitzKaup-Newell-Segur [1] system of equations

$$
\begin{aligned}
\Phi_{x}^{(2)}+\mathrm{i} k \sigma_{3} \Phi^{(2)} & =Q(x, t) \Phi^{(2)}, \quad x \in \mathbb{R}, \quad t>0, \\
\Phi_{t}^{(2)}+4 \mathrm{i} k^{3} \sigma_{3} \Phi^{(2)} & =\hat{Q}(x, t, k) \Phi^{(2)},
\end{aligned}
$$

where

$$
\begin{aligned}
Q(x, t) & =\left(\begin{array}{cc}
0 & q(x, t) \\
-\bar{q}(x, t) & 0
\end{array}\right), \\
\hat{Q}(x, t, k) & =2 k Q(x, t)-\mathrm{i}\left(Q^{2}(x, t)+Q_{x}(x, t)\right) \sigma_{3}
\end{aligned}
$$

with the function $q(x, t)$ given by

$$
q(x, t)=2 \mathrm{i} \lim _{k \rightarrow \infty} k\left[M^{(2)}(x, t, k)\right]_{12}=\frac{-1}{\pi} \int_{\Gamma^{(2)}}\left(\left[I+N^{(2)}(x, t, k)\right]\left[I-J^{(2)}(x, t, k)\right]\right)_{12} \mathrm{~d} k .
$$

Corollary 1. The function $q(x, t)$ is smooth for $x \in \mathbb{R}, t \neq 0$, and it satisfies the $N L S$ equation (1.1)

Proof. The matrix $\Phi^{(2)}(x, t, k)$ is analytic in $k \in \mathbb{C} \backslash \Gamma^{(2)}$. It has the following jump across $\Gamma^{(2)}$.

$$
\Phi_{-}^{(2)}(x, t, k)=\Phi_{+}^{(2)}(x, t, k) J_{0}^{(2)}(k), \quad k \in \Gamma^{(2)},
$$

where $J_{0}^{(2)}(k)=\mathrm{e}^{\left(\mathrm{i} k x+2 \mathrm{i} k^{2} t\right) \sigma_{3}} J^{(2)}(x, t, k) \mathrm{e}^{\left(-\mathrm{i} k x-2 \mathrm{i} k^{2} t\right) \sigma_{3}}$ is independent on $x$ and $t$. By differentiation with respect to $x$ and $t$ we get

$$
\begin{aligned}
& \frac{\partial \Phi_{-}^{(2)}(x, t, k)}{\partial x}\left(\Phi_{-}^{(2)}(x, t, k)\right)^{-1}=\frac{\partial \Phi_{+}^{(2)}(x, t, k)}{\partial x}\left(\Phi_{+}^{(2)}(x, t, k)\right)^{-1} \\
& \frac{\partial \hat{\Phi}_{-}^{(1)}(x, t, k)}{\partial t}\left(\Phi_{-}^{(2)}(x, t, k)\right)^{-1}=\frac{\partial \Phi_{+}^{(2)}(x, t, k)}{\partial t}\left(\Phi_{+}^{(2)}(x, t, k)\right)^{-1}
\end{aligned}
$$

for $k \in \Gamma^{(2)}$. The last relations mean that the matrix logarithmic derivatives $\Phi_{x}^{(2)}(x, t, k)\left(\Phi^{(2)}(x, t, k)\right)^{-1}$ and $\Phi_{t}^{(2)}(x, t, k)\left(\Phi^{(2)}(x, t, k)\right)^{-1}$ are analytic (entire) in $k \in \mathbb{C}$. The Cauchy integral for $M^{(2)}(x, t, k)$ gives the following asymptotic formulas:

$$
\begin{gathered}
M^{(2)}(x, t, k)=I+\frac{m^{(2)}(x, t)}{k}+\mathrm{O}\left(k^{-2}\right), \\
\frac{\Phi^{(2)}(x, t, k)}{\partial x}=\frac{m_{x}^{(2)}(x, t)}{k}+\mathrm{O}\left(k^{-2}\right), k \rightarrow \infty,
\end{gathered}
$$


where

$$
m^{(2)}(x, t)=\frac{\mathrm{i}}{2 \pi} \int_{\Gamma^{(2)}}\left[I+N^{(2)}(x, t, k)\right]\left[I-J^{(2)}(x, t, k)\right] \mathrm{d} k .
$$

Hence

$$
\Phi_{x}^{(2)}(x, t, k)\left(\Phi^{(2)}(x, t, k)\right)^{-1}=-\mathrm{i} k \sigma_{3}+\mathrm{i}\left[\sigma_{3}, m^{(1)}\right]+\mathrm{O}\left(k^{-1}\right), k \rightarrow \infty .
$$

Here $[A, B]=A B-B A$. Therefore, by Liouville's theorem, the logarithmic derivative $\Phi_{x}^{(2)}(x, t, k)\left(\Phi^{(2)}(x, t, k)\right)^{-1}$ is a polynomial of degree 1 in $k$ :

$$
U(k):=\Phi_{x}^{(2)}(x, t, k)\left(\hat{\Phi}^{(1)}(x, t, k)\right)^{-1}=-\mathrm{i} k \sigma_{3}+Q(x, t),
$$

where $Q(x, t) \equiv \mathrm{i}\left[\sigma_{3}, m^{(2)}\right]=\left(\begin{array}{cc}0 & q(x, t) \\ p(x, t) & 0\end{array}\right)$. The symmetry of the contour and the jump matrix ensure the symmetry of the matrices $\sigma_{2} \overline{\Phi^{(2)}(x, t, k)} \sigma_{2}=\Phi^{(2)}(x, t, k)$ and $\sigma_{2} \bar{U}(\bar{k}) \sigma_{2}=U(k)$, where $\sigma_{2}=\left(\begin{array}{cc}0 & -\mathrm{i} \\ \mathrm{i} & 0\end{array}\right)$. Therefore $Q(x, t)=-Q^{*}(x, t)$ is anti-Hermitian, i.e. $p(x, t)=-\bar{q}(x, t)$. Thus $\Phi^{(2)}(x, t, k)$ satisfies the equation (4.10) and a scalar function $q(x, t)$ is defined by (4.12). The function $q(x, t)$ is smooth in $x \in \mathbb{R}, t \neq 0$, because the jump matrix $J^{(2)}(x, t, k)$ is smooth in $x$ and $t$ by definition, the function $N^{(2)}(x, t, k)$ is smooth by Theorem 5 , and the integrals that have presented any partial derivative with respect to $x$ and $t$ of the function $q(x, t)$ are well convergent under the condition $t \neq 0$.

In the same way as before, we find that $\Phi_{t}^{(2)}(x, t, k)\left(\Phi^{(2)}(x, t, k)\right)^{-1}$ is also a polynomial,

$$
\Phi_{t}^{(2)}(x, t, k)\left(\Phi^{(2)}(x, t, k)\right)^{-1}=-2 \mathrm{i} k^{2} \sigma_{3}+k Q_{1}(x, t)+Q_{0}(x, t) .
$$

Thus we see that the matrix $\Phi^{(2)}(x, t, k)$ satisfies two differential equations:

$$
\begin{gathered}
\Phi_{x}^{(2)}(x, t, k)=\left(Q(x, t)-\mathrm{i} k \sigma_{3}\right) \Phi^{(2)}(x, t, k), \\
\Phi_{t}^{(2)}(x, t, k)=\left(Q_{0}(x, t)+k Q_{1}(x, t)-2 \mathrm{i} k^{2} \sigma_{3}\right) \Phi^{(2)}(x, t, k) .
\end{gathered}
$$

Their compatibility $\left(\Phi_{x t}^{(2)}(x, t, k)=\Phi_{t x}^{(2)}(x, t, k)\right)$ gives the system of matrix equations:

$$
\begin{gathered}
{\left[\sigma_{3}, Q_{1}\right]+2\left[Q, \sigma_{3}\right]=0,} \\
\left(Q_{1}\right)_{x}+\mathrm{i}\left[\sigma_{3}, Q_{0}\right]-\left[Q, Q_{1}\right]=0, \\
Q_{t}-\left(Q_{0}\right)_{x}+\left[Q, Q_{0}\right]=0 .
\end{gathered}
$$

The first and the second equations give $Q_{1}=2 Q$, one $Q_{0}=-\mathrm{i} \alpha \sigma_{3}-\mathrm{i} Q_{x} \sigma_{3}$ while the third matrix equation defines $\alpha=Q^{2}(x, t)=-|q(x, t)|^{2} I$ and NLS equation in the matrix form:

$$
\mathrm{i} Q_{t}-Q_{x x} \sigma_{3}+2 Q^{3} \sigma_{3}=0 .
$$

Thus the theorem is proved.

Corollary 2. Let $\Phi(x, t, k):=M(x, t, k) \mathrm{e}^{\left(\mathrm{i} k x+2 \mathrm{i} k^{2} t\right) \sigma_{3}}$, where $M(x, t, k)$ is defined by the original $R H$ problem (i) - (iv). Then the matrix $\Phi(x, t, k)$ satisfies the equations (4.10), (4.11) with the matrix

$$
Q(x, t)=\mathrm{i}\left[\sigma_{3}, m(x, t)\right]
$$

where

$$
m(x, t)=\lim _{k \rightarrow \infty} k(M(x, t, k)-I)=\lim _{k \rightarrow \infty} k\left(M^{(2)}(x, t, k)-I\right)=m^{(2)}(x, t)
$$


Proof. Since the matrix $M(x, t, k)$ is smooth in $x \in \mathbb{C}, t \neq 0$, and solves the original $\mathrm{RH}$ problem, then in the same way as in the theorem (6), we prove that the matrix $\Phi$ satisfies the equations (4.10), (4.11) with the matrix $Q(x, t)=\mathrm{i}\left[\sigma_{3}, m(x, t)\right]$. By the definition, we have

$$
\begin{aligned}
m(x, t) & =\lim _{k \rightarrow \infty} k(M(x, t, k)-I) \\
& =\lim _{k \rightarrow \infty} k\left(M^{(2)}(x, t, k)-I\right)\left(G^{(1)}(x, t, k)\right)^{-1} \delta^{\sigma_{3}}(k, \xi) \\
& +k\left(\left(\hat{G}^{(1)}(x, t, k)\right)^{-1} \delta^{\sigma_{3}}(k, \xi)-I\right) \\
& =\lim _{k \rightarrow \infty} k\left(M^{(2)}(x, t, k)-I\right)=m^{(2)}(x, t)
\end{aligned}
$$

because $\lim _{k \rightarrow \infty}\left(\left(G^{(1)}(x, t, k)\right)^{-1} \delta^{\sigma_{3}}(k, \xi)-I\right)=0$.

In the previous sections we construct the set of matrix-valued Riemann - Hilbert problems (i)-(iv). We prove the existence of solutions to these problems, continuity in the parameters $(x, t) \in \mathbb{R} \times \mathbb{R}$ and smoothness for $x \in \mathbb{R}$ and $t \neq 0$. Any solution of the RH problem (i)-(iv) is associated with the function $q(x, t)$ by the formula $q(x, t)=\lim _{k \rightarrow \infty} 2 \mathrm{i} k M(x, t, k)_{21}$. We prove that $q(x, t)$ is smooth in $x \in \mathbb{R}, t \neq 0$ and satisfies the focusing nonlinear Schrödunger equation (1.1). The behavior of $q(x, t)$ in the neighborhood of $t=0$ is determined by the parameter of the original $\mathrm{RH}$ problem function $r($.$) . If r(.) \in L_{1}(\Gamma)$, then $q(x, t)$ is jointly continuous in $(x, t) \in \mathbb{R} \times \mathbb{R}$. If $r(.) \notin L_{1}(\Gamma)$, then the integral $\int_{\Gamma} r(s) \mathrm{e}^{2 \mathrm{i} s x+8 i s^{3} t} \mathrm{~d} s$ converges improperly for $x, t \neq 0$ and

$$
q(x, t)+\frac{1}{\pi} \int_{\Gamma} r(s) \mathrm{e}^{2 \mathrm{i} s x+8 \mathrm{i} s^{3} t} \mathrm{~d} s \in C(\mathbb{R} \times \mathbb{R}) .
$$

\section{$5 \quad$ Long-time asymptotics}

Remark. In the present paper, we consider the initial value problem with the pure step-like initial conditions. This is done basically to fix ideas while avoiding technical complications arising in the case when $q_{0}(x)$ given by $(1.2)$ is not exact initial data but the large- $x$ asymptotics for $q(x, 0)$. In the latter case, the reflection coefficient $r(k)$ cannot, in general, be analytically extended from the real axis, which leads to additional steps in the sequence of $\mathrm{RH}$ problem deformations related to rational approximations of $r(k)$ and the consequent "opening of lenses" procedures (cf. [14], [18], [8]) along the real axis and the contour $\Sigma$. Also, possible zeros of the spectral function $a(k)$ will generate solitons.

\subsection{Region $\xi \equiv \frac{x}{4 t}=-\operatorname{Re} E_{0}+\frac{\rho \ln t}{4 \operatorname{Im} E_{0} t}$.}

In this region we continue to use the function $\theta(k, \xi)=2 k^{2}+4 \xi k$. Let us pick up a sufficiently small but fixed positive number $r>0$. First of all, we 'bend' the segment $\left[E_{0}, \overline{E_{0}}\right]$ to the right, and call the resulting (smooth) line

$$
\hat{\Sigma}_{0}=\hat{\Sigma}_{0}^{+} \cup \hat{\Sigma}_{0}^{-}, \quad \text { where } \quad \hat{\Sigma}_{0}^{+}=\left[E_{0}, E_{0}+r, \operatorname{Re} E_{0}+10 r\right], \quad \hat{\Sigma}_{0}^{-}=\left[\operatorname{Re} E_{0}+10 r, \overline{E_{0}}+r, \overline{E_{0}}\right] .
$$

Denote by $\Omega_{u}$ the domain between $\mathbb{R}, \Sigma_{0}^{+}$, and $\hat{\Sigma}_{0}^{+}$, and denote by $\Omega_{d}$ the domain between $\mathbb{R}, \Sigma_{0}^{-}, \hat{\Sigma}_{0}^{-}$ (subscript $u$ means 'up', $d$ means 'down'). Denote by $\hat{\Sigma}$ the contour $\Sigma$, where instead of the segment $\Sigma_{0}$ we take the curve $\hat{\Sigma}_{0}$,

$$
\hat{\Sigma}=\hat{\Sigma}_{0} \cup \ldots \cup \Sigma_{j} \cup \ldots \cup \Sigma_{n} .
$$


Function $f(k)$, defined on $k \in\left[E_{0}, \bar{E}_{0}\right]$, admits a piece-wise analytic continuation $\widehat{f}(k)$ in a neighborhood of the segment $\left[E_{0}, 0\right]$,

$$
\widehat{f}(k+0)=f(k), \quad k \in\left[E_{0}, 0\right],
$$

where $\widehat{f}(k)$ is analytic in a neighborhood of the segment $\left[E_{0}, 0\right]$, excluding the segment $\left[E_{0}, 0\right]$ itself. Furthermore, define

$$
\begin{aligned}
M^{(2)}(\xi, t ; k) & =M(\xi, t ; k)\left[\begin{array}{cc}
1 & 0 \\
\widehat{f}(k) \mathrm{e}^{2 \mathrm{i} t \theta(k, \xi)} & 1
\end{array}\right], k \in \Omega_{u}, \\
& =M(\xi, t ; k)\left[\begin{array}{cc}
1 & -\overline{\widehat{f}(\bar{k})} \mathrm{e}^{-2 \mathrm{i} t \theta(k, \xi)} \\
0 & 1
\end{array}\right], k \in \Omega_{d}, \\
& =M(\xi, t ; k), \text { elsewhere. }
\end{aligned}
$$

The function $M^{(2)}$ satisfies the Basic RH problem with the contour $\hat{\Sigma}$ instead of $\Sigma$ (see Figure 4).

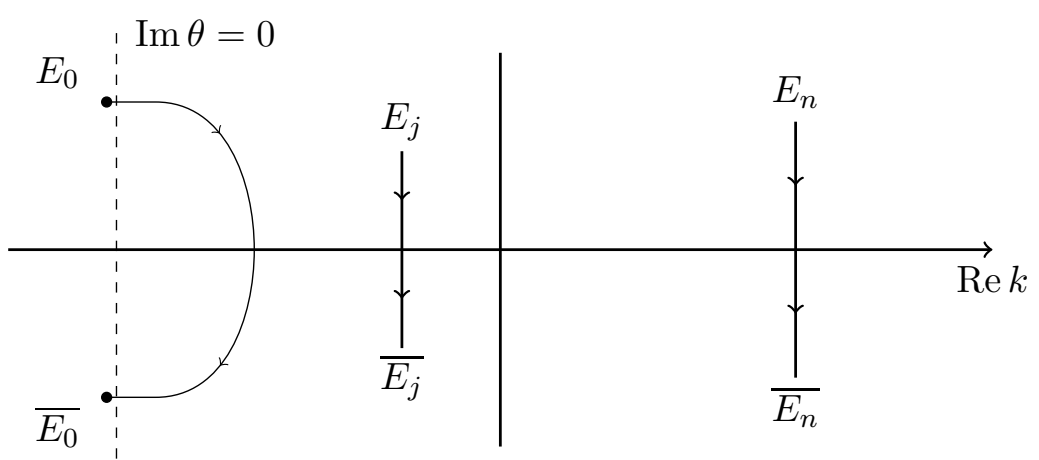

Figure 4: The oriented contour $\hat{\Gamma}=\mathbb{R} \cup \hat{\Sigma}$ and (dashed) line $\operatorname{Im} \theta=0$.

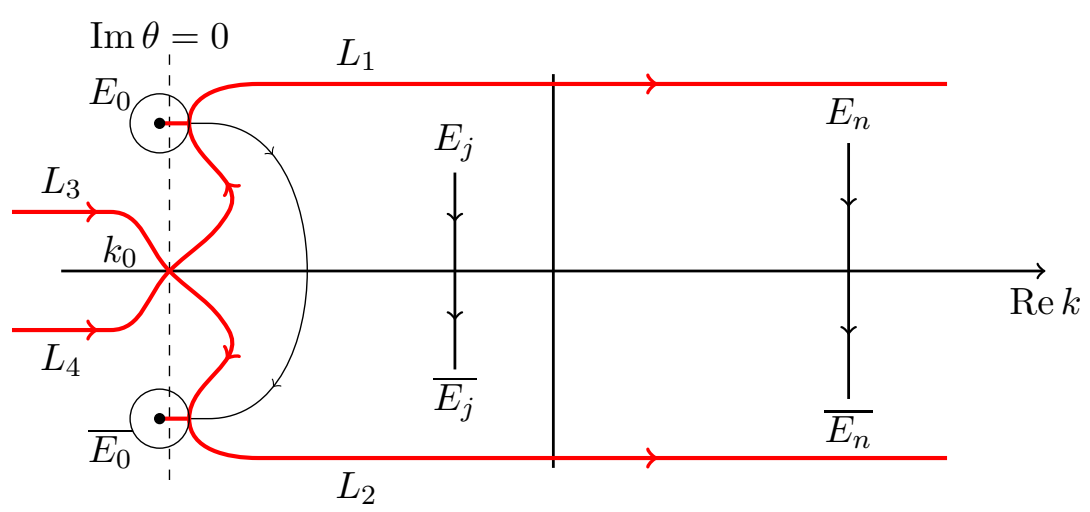

Figure 5: Opening of the lenses. The point $k_{0}=k_{0}(\xi)=-\xi$.

We draw the lines $L_{j}, j=1,2,3,4$, as shown in Figure 5 Denote the domain consisted between $L_{1}$ and $\mathbb{R}$ by $\Omega_{1}$, the one between $L_{2}$ and $\mathbb{R}$ by $\Omega_{2}$, the one between $L_{3}$ and $\mathbb{R}$ by $\Omega_{3}$, and the domain between $L_{4}$ and $\mathbb{R}$ by $\Omega_{4}$. Furthermore, introduce the scalar function $\delta(k, \xi)$ as the solution to the following conjugation problem:

$$
\begin{aligned}
& \delta_{+}(k, \xi)=\delta_{-}(k, \xi)\left(1+|r(k)|^{2}\right), k \in\left(-\infty, k_{0}=-\xi\right), \\
& \lim _{k \rightarrow \infty} \delta(k)=1, \\
& \delta(k) \text { is analytic in } k \in \mathbb{C} \backslash\left(-\infty, k_{0}\right] .
\end{aligned}
$$


The function $\delta(k, \xi)$ satisfies the symmetry property

$$
\bar{\delta}(\bar{k}, \xi) \cdot \delta(k, \xi)=1
$$

and can be found explicitly in the form

$$
\delta(k, \xi)=\exp \left[\frac{1}{2 \pi \mathrm{i}} \int_{-\infty}^{k_{0}} \frac{\ln \left(1+|r(s)|^{2}\right) \mathrm{d} s}{s-k}\right] .
$$

Furthermore, define the function

$$
\begin{aligned}
M^{(3)}(\xi, t ; k) & =M^{(2)}(\xi, t ; k)\left[\begin{array}{cc}
1 & 0 \\
-r(k) \delta^{-2}(k, \xi) \mathrm{e}^{2 \mathrm{i} t \theta(k, \xi)} & 1
\end{array}\right], k \in \Omega_{1}, \\
& =M^{(2)}(\xi, t ; k)\left[\begin{array}{cc}
1 & \overline{r(\bar{k})} \delta^{2}(k, \xi) \mathrm{e}^{-2 \mathrm{i} t \theta(k, \xi)} \\
0 & 1
\end{array}\right], k \in \Omega_{2}, \\
& =M^{(2)}(\xi, t ; k)\left[\begin{array}{cr}
1 & -\overline{r(\bar{k})}(1+r(k) \overline{r(\bar{k})})^{-1} \delta^{2}(k, \xi) \mathrm{e}^{-2 \mathrm{i} t \theta(k, \xi)} \\
0 & 1
\end{array}\right], k \in \Omega_{3}, \\
& =M^{(2)}(\xi, t ; k)\left[\begin{array}{rr}
1 \\
r(k)(1+r(k) \overline{r(\bar{k})})^{-1} \delta^{-2}(k, \xi) \mathrm{e}^{2 \mathrm{i} t \theta(k, \xi)} & 1
\end{array}\right], k \in \Omega_{4},
\end{aligned}
$$

In view of (4.6), the function $M^{(3)}$ satisfies the following RHP:

Transformed RH problem. Find a $2 \times 2$-valued function $M^{(3)}(\xi, t ; k)$ such that

(i) $M^{(3)}(\xi, t, k)$ is analytic in $k \in \mathbb{C} \backslash \tilde{\Gamma}, \tilde{\Gamma}=L_{1} \cup L_{2} \cup L_{3} \cup L_{4} \cup\left[E_{0}, E_{0}+r\right] \cup\left[\overline{E_{0}}, \overline{E_{0}}+r\right]$.

(ii) the boundary values $M_{ \pm}^{(3)}(x, t, k)$ satisfy the jump conditions

$$
M_{-}^{(3)}(x, t, k)=M_{+}^{(3)}(x, t, k) J^{(3)}(x, t, k), k \in \tilde{\Gamma},
$$

where the jump matrix $J^{(3)}(x, t, k)$ is defined as follows:

$$
\begin{aligned}
J^{(3)}(x, t, k) & =\left[\begin{array}{cc}
1 & 0 \\
-r(k) \delta^{-2}(k, \xi) \mathrm{e}^{2 \mathrm{i} t \theta(k, \xi)} & 1
\end{array}\right], k \in L_{1}, \\
& =\left[\begin{array}{cc}
1 & -\overline{r(\bar{k})} \delta^{2}(k, \xi) \mathrm{e}^{-2 \mathrm{i} t \theta(k, \xi)} \\
0 & 1
\end{array}\right], k \in L_{2}, \\
& =\left[\begin{array}{cc}
1 & -\overline{r(\bar{k})}(1+r(k) \overline{r(\bar{k})})^{-1} \delta^{2}(k, \xi) \mathrm{e}^{-2 \mathrm{i} t \theta(k, \xi)} \\
0 & 1
\end{array}\right], k \in L_{3}, \\
& =\left[\begin{array}{cc}
1 \\
-r(k)(1+r(k) \overline{r(\bar{k})})^{-1} \delta^{-2}(k, \xi) \mathrm{e}^{2 \mathrm{i} t \theta(k, \xi)} & 1
\end{array}\right], k \in L_{4},
\end{aligned}
$$




$$
\begin{aligned}
& =\left[\begin{array}{cc}
1 & 0 \\
\widehat{f}(k) \delta(k, \xi)^{-2} \mathrm{e}^{2 \mathrm{i} t \theta(k, \xi)} & 1
\end{array}\right], k \in l^{+}\left(\sim\left[E_{0}, E_{0}+r\right]\right), \\
& =\left[\begin{array}{cc}
1 & -\overline{\widehat{f}(\bar{k})} \delta(k, \xi)^{2} \mathrm{e}^{-2 \mathrm{i} t \theta(k, \xi)} \\
0 & 1
\end{array}\right], k \in l^{-}\left(\sim\left[\overline{E_{0}}+r, \overline{E_{0}}\right]\right),
\end{aligned}
$$

(iii)

$$
M^{(3)}(\xi, t ; k)=I+\mathrm{O}\left(\frac{1}{k}\right) \quad \text { as } k \rightarrow \infty .
$$

Let us notice, that the jumps on $L_{j}, j=1, \ldots, 4$ except for the vicinity of the point $k_{0}$, are exponentially small, and hence the main contribution comes from the segments $l^{+} \sim\left[E_{0}, E_{0}+r\right]$, $l^{-} \sim\left[\overline{E_{0}}, \overline{E_{0}+r}\right]$ and (sub-leading of order $t^{-1 / 2}$ ) contribution comes from the neighborhood of $k_{0}$.

\subsubsection{Local changes of variables in the vicinity of the points $k=E_{0}, k=\bar{E}_{0}$.}

Let us denote $E_{0}=A+\mathrm{i} B$, and take

$$
\xi=-A-\frac{\rho}{4 B} \frac{\ln t}{t}
$$

and make local changes of variable

$$
k=E_{0}+y, \quad k=\overline{E_{0}}+y_{d}
$$

in the vicinity of the points $k=E_{0}, \overline{E_{0}}$, respectively. Let us notice, that the behavior of $f(k)$ on the interval $k \in\left(E_{0}, E_{0}-\mathrm{i} r\right)$ is as follows:

$$
f(k)=\mathrm{e}^{-\pi \mathrm{i} / 4} c_{0} \sqrt{\mathrm{i}\left(k-E_{0}\right)}\left(1+\mathcal{O}\left(\sqrt{\mathrm{i}\left(k-E_{0}\right)}\right)\right), \quad k \in\left(E_{0}, E_{0}-\mathrm{i} r\right),
$$

where the root is positive on $\left(E_{0}, E_{0}-\mathrm{i} r\right)$. Hence, the behavior of the function $\widehat{f}(k)$ in the vicinity of the point $k=E_{0}$ is as follows:

$$
\widehat{f}(k)=\sqrt{k-E_{0}} \cdot \hat{\phi}(k), \quad \overline{\widehat{f}(\bar{k})}=\sqrt{k-\overline{E_{0}}} \cdot \overline{\hat{\phi}(\bar{k})}, \quad \hat{\phi}\left(k=E_{0}\right)=c_{0} .
$$

where $\phi(k)$ is an analytic function in a vicinity of $k=E_{0}$, separated both from 0 and $\infty$.

We have furthermore

$$
\frac{\widehat{f}(k) \mathrm{e}^{2 \mathrm{i} t \theta(k, \xi)}}{\delta(k, \xi)^{2}}=-\sqrt{t z} \mathrm{e}^{-t z} t^{2 \gamma} \mathrm{e}^{-2 \mathrm{i} \varphi(t)}(-\phi(k, \xi)), \quad \frac{-\bar{f}(\bar{k})}{\mathrm{e}^{2 \mathrm{i} t \theta(k, \xi)}}=\sqrt{t z_{d}} \mathrm{e}^{-t z_{d}} t^{2 \gamma} \mathrm{e}^{2 \mathrm{i} \varphi(t)}(-\overline{\phi(\bar{k})})
$$

where we made the further local change of variables

$$
z=y\left(8 B+\frac{2 \mathrm{i} \rho \ln t}{B t}\right)-4 \mathrm{i} y^{2}, \quad z_{d}=y_{d}\left(8 B-\frac{2 \mathrm{i} \rho \ln t}{B t}\right)+4 \mathrm{i} y_{d}^{2},
$$

and denoted

$\gamma=\rho-\frac{1}{4}, \quad \varphi(t)=2 t\left(A^{2}+B^{2}\right)+\frac{\rho A \ln t}{B}, \quad \phi(k, \xi)=\sqrt{\frac{y}{z}} \cdot \frac{\hat{\phi}(k)}{\delta(k, \xi)^{2}}, \quad \overline{\phi(\bar{k}, \xi)}=\sqrt{\frac{y_{d}}{z_{d}}} \cdot \overline{\hat{\phi}(\bar{k})} \cdot \delta(k, \xi)^{2}$.

We fix the segments $l^{ \pm}$by the condition that $z, z_{d}$ are real on $l^{ \pm}$, respectively. Finally, denote

$$
\zeta=z t, \quad \zeta_{d}=z_{d} t
$$


Hence, the jump $J^{(3)}$ on the segment $l^{+}$can be written as

$$
\begin{aligned}
J^{(3)} & =t^{-\gamma \sigma_{3}} \mathrm{e}^{\mathrm{i} \varphi(t) \sigma_{3}}(-\phi(k, \xi))^{-\frac{\sigma_{3}}{2}}\left[\begin{array}{cc}
1 & 0 \\
-\sqrt{\zeta} \mathrm{e}^{-\zeta} & 1
\end{array}\right](-\phi(k, \xi))^{\frac{\sigma_{3}}{2}} \mathrm{e}^{-\mathrm{i} \varphi(t) \sigma_{3}} t^{\gamma \sigma_{3}}, \quad k \in l^{+}, \\
& =t^{\gamma \sigma_{3}} \mathrm{e}^{\mathrm{i} \varphi(t) \sigma_{3}}[-\overline{\phi(\bar{k}, \xi)}]^{\frac{\sigma_{3}}{2}}\left[\begin{array}{cc}
1 & \sqrt{\zeta_{d}} \mathrm{e}^{-\zeta_{d}} \\
0 & 1
\end{array}\right][-\overline{\phi(\bar{k}, \xi)}]^{-\frac{\sigma_{3}}{2}} \mathrm{e}^{-\mathrm{i} \varphi(t) \sigma_{3}} t^{-\gamma \sigma_{3}}, \quad k \in l^{-} .
\end{aligned}
$$

Formula (5.3) clearly indicates the possibility of using generalized Laguerre polynomials of index $1 / 2$, which will be done in the next section.

\subsubsection{Generalized Laguerre polynomials of index $\frac{1}{2}$.}

Denote $p_{n}(\zeta)=L_{n}^{(1 / 2)}(\zeta)=\frac{(-1)^{n}}{n !} \zeta^{n}+\ldots, \quad \pi_{n}(\zeta)=(-1)^{n} n ! p_{n}(\zeta)=\zeta^{n}+\ldots$

$$
\int_{0}^{+\infty} \zeta^{1 / 2} \mathrm{e}^{-\zeta} p_{n}(\zeta) p_{m}(\zeta) d \zeta=\frac{\Gamma\left(n+\frac{3}{2}\right)}{n !} \delta_{m, n}, \int_{0}^{+\infty} \zeta^{1 / 2} \mathrm{e}^{-\zeta} \pi_{n}(\zeta) \pi_{m}(\zeta) d \zeta=\Gamma\left(n+\frac{3}{2}\right) n ! \delta_{m, n}
$$

The generalized Laguerre polynomials with index $\frac{1}{2}$ and degree $n$ solve a RHP of the form

$$
\begin{aligned}
L_{-}(\zeta) & =L_{+}(\zeta) J_{L}(\zeta), \quad \zeta \in \mathbb{R}_{+}, \\
J_{L}(\zeta) & =\left(\begin{array}{cc}
1 & 0 \\
-\sqrt{\zeta} \mathrm{e}^{-\zeta} & 1
\end{array}\right), \\
L(\zeta) & =\left(\mathbf{1}+\mathcal{O}\left(\zeta^{-1}\right)\right) \zeta^{-n \sigma_{3}}, \quad \zeta \rightarrow \infty .
\end{aligned}
$$

and the solution is written as follows: for $n \geq 1$

$$
L(\zeta)=\left(\begin{array}{cc}
\frac{-2 \pi \mathrm{i}}{\Gamma\left(n+\frac{1}{2}\right) \Gamma(n)} \frac{1}{2 \pi \mathrm{i}} \int_{0}^{+\infty} \frac{\sqrt{s} \mathrm{e}^{-s} \pi_{n-1}(s) \mathrm{d} s}{s-\zeta} & \frac{-2 \pi \mathrm{i}}{\Gamma\left(n+\frac{1}{2}\right) \Gamma(n)} \pi_{n-1}(\zeta) \\
\frac{1}{2 \pi \mathrm{i}} \int_{0}^{+\infty} \frac{\sqrt{s} \mathrm{e}^{-s} \pi_{n}(s) \mathrm{d} s}{s-\zeta} & \pi_{n}(\zeta)
\end{array}\right),
$$

and for $n=0$

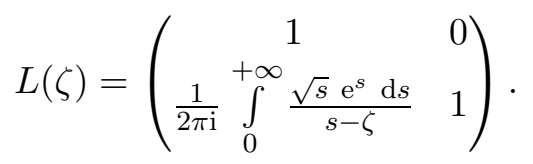

Furthermore, the matrix function

$$
L_{d}(\zeta)=\left(\begin{array}{ll}
0 & 1 \\
1 & 0
\end{array}\right) L(\zeta)\left(\begin{array}{ll}
0 & 1 \\
1 & 0
\end{array}\right)
$$

solves a RHP of the form

$$
\begin{aligned}
L_{d,-}(\zeta) & \left.=L_{d,+}(\zeta) J_{L_{d}}(\zeta), \quad \zeta \in(+\infty, 0) \text { (the orientation is from }+\infty \text { to } 0\right), \\
J_{L_{d}}(\zeta) & =\left(\begin{array}{cc}
1 & \sqrt{\zeta_{d}} \mathrm{e}^{-\zeta_{d}} \\
0 & 1
\end{array}\right), \\
L_{d}\left(\zeta_{d}\right) & =\left(\mathbf{1}+\mathcal{O}\left(\zeta_{d}^{-1}\right)\right) \zeta_{d}^{n \sigma_{3}}, \quad \zeta_{n} \rightarrow \infty .
\end{aligned}
$$


To show the relation with our RH problem for $M^{(3)}$, consider the functions

$$
\begin{aligned}
& L^{(1)}=\left(-\phi(k, \xi) t^{2 \gamma} \mathrm{e}^{-2 \mathrm{i} \varphi(t)}\right)^{-\sigma_{3} / 2} \cdot L(\zeta) \cdot\left(-\phi(k, \xi) t^{2 \gamma} \mathrm{e}^{-2 \mathrm{i} \varphi(t)}\right)^{\sigma_{3} / 2} \\
& L_{d}^{(1)}=\left(-\bar{\phi}(\bar{k}, \xi) t^{2 \gamma} \mathrm{e}^{2 \mathrm{i} \varphi(t)}\right)^{\sigma_{3} / 2} \cdot L_{d}\left(\zeta_{d}\right) \cdot\left(-\bar{\phi}(\bar{k}, \xi) t^{2 \gamma} \mathrm{e}^{2 \mathrm{i} \varphi(t)}\right)^{-\sigma_{3} / 2}
\end{aligned}
$$

they have the following jumps on $\zeta \in(0,+\infty), \zeta_{d} \in(+\infty, 0)$, respectively (compare with (5.3)):

$$
\left(L_{+}^{(1)}\right)^{-1} L_{-}^{(1)}=\left(\begin{array}{cc}
1 & 0 \\
\phi(k, \xi) t^{2 \gamma} \mathrm{e}^{-2 \mathrm{i} \varphi} \sqrt{\zeta} \mathrm{e}^{-\zeta} & 1
\end{array}\right), \quad\left(L_{d,+}^{(1)}\right)^{-1} L_{d,-}^{(1)}=\left(\begin{array}{cc}
1 & -\bar{\phi}(\bar{k}, \xi) t^{2 \gamma} \mathrm{e}^{2 \mathrm{i} \varphi} \sqrt{\zeta_{d}} \mathrm{e}^{-\zeta_{d}} \\
0 & 1
\end{array}\right) .
$$

Further, developing up to $\zeta^{-1}, \zeta_{d}^{-1}$ term in the asymptotics (5.4), (5.6) of $L, L_{d}$ as $\zeta, \zeta_{d} \rightarrow \infty$, we obtain

$$
\begin{gathered}
L(\zeta)=\left[\left(\begin{array}{cc}
1+\frac{n^{2}+\frac{n}{2}}{\zeta} & \frac{-2 \pi \mathrm{i} n}{\Gamma\left(n+\frac{1}{2}\right) n ! \zeta} \\
\frac{-n ! \Gamma\left(n+\frac{3}{2}\right)}{2 \pi \mathrm{i} \zeta} & 1-\frac{n^{2}+\frac{n}{2}}{\zeta}
\end{array}\right)+\mathrm{O}\left(\zeta^{-2}\right)\right] \zeta^{-n \sigma_{3}}, \quad \zeta \rightarrow \infty, \\
L_{d}(\zeta)=\left[\left(\begin{array}{cc}
1-\frac{n^{2}+\frac{n}{2}}{\zeta_{d}} & \frac{-n ! \Gamma\left(n+\frac{3}{2}\right)}{2 \pi \mathrm{i} \zeta_{d}} \\
\frac{-2 \pi \mathrm{i} n}{\Gamma\left(n+\frac{1}{2}\right) n ! \zeta_{d}} & 1+\frac{n^{2}+\frac{n}{2}}{\zeta_{d}}
\end{array}\right)+\mathrm{O}\left(\zeta_{d}^{-2}\right)\right] \zeta_{d}^{n \sigma_{3}}, \quad \zeta_{d} \rightarrow \infty .
\end{gathered}
$$

The formulas (5.7), (5.8) include also the case $n=0$.

\subsubsection{Local change of variable in the vicinity of the point $k=k_{0} \equiv-\xi$.}

For $k$ in the vicinity the point $k=k_{0} \equiv-\xi$ we make the following changes of variable:

$$
k=k_{0}+\mu, \quad \lambda=\sqrt{2 t} \mu .
$$

Then

$$
\theta(k, \xi)=2 k^{2}+4 \xi k=-2 \xi^{2}+2 \mu^{2}, \quad 2 \mathrm{i} t \theta(k, \xi)=-4 \mathrm{i} t \xi^{2}+2 \mathrm{i} \lambda^{2} .
$$

Furthermore, the function $\delta(k, \xi)$ can be rewritten in the form

$$
\delta(k, \xi)=\left(k-k_{0}\right)^{-\mathrm{i} \nu(\xi)} \cdot \chi(k, \xi), \quad \text { where } \quad \nu=\frac{1}{2 \pi} \ln \left(1+\left|r\left(k_{0}\right)\right|^{2}\right),
$$

and the function $\chi(k, \xi)$ has a non-zero limit at the point $k=k_{0}$,

$$
\chi(k, \xi)=(k+N)^{\mathrm{i} \nu} \cdot \exp \left[\frac{1}{2 \pi \mathrm{i}} \int_{-N}^{k_{0}} \frac{\ln \frac{1+|r(s)|^{2}}{1+\left|r\left(k_{0}\right)\right|^{2}} \mathrm{~d} s}{s-k}+\frac{1}{2 \pi \mathrm{i}} \int_{-\infty}^{-N} \frac{\ln \left(1+|r(s)|^{2}\right) \mathrm{d} s}{s-k}\right] .
$$

The latter expression does not depend on the choice of the parameter $-N<k_{0}$.

Let us pick up some sufficiently small (fixed) positive $\delta>0$. Then the jump matrix $J^{(3)}$ has the following representation for $\left|k-k_{0}\right|<\delta$ :

$$
\begin{aligned}
J^{(3)}= & \left(\chi(k, \xi) \mathrm{e}^{2 \mathrm{i} t \xi^{2}}(2 t)^{\frac{\mathrm{i} \nu}{2}}\right)^{\sigma_{3}}\left[\begin{array}{ccc}
1 & 0 \\
-r(k) \lambda^{2 \mathrm{i} \nu} \mathrm{e}^{2 \mathrm{i} \lambda^{2}} & 1
\end{array}\right]\left(\chi(k, \xi) \mathrm{e}^{2 \mathrm{i} t \xi^{2}}(2 t)^{\frac{\mathrm{i} \nu}{2}}\right)^{-\sigma_{3}}, k \in L_{1}, \\
& \left(\chi(k, \xi) \mathrm{e}^{2 \mathrm{i} t \xi^{2}}(2 t)^{\frac{\mathrm{i} \nu}{2}}\right)^{\sigma_{3}}\left[\begin{array}{ccc}
1 & -\overline{r(\bar{k})} \lambda^{-2 \mathrm{i} \nu} \mathrm{e}^{-2 \mathrm{i} \lambda^{2}} \\
0 & 1
\end{array}\right]\left(\chi(k, \xi) \mathrm{e}^{2 \mathrm{i} t \xi^{2}}(2 t)^{\frac{\mathrm{i} \nu}{2}}\right)^{-\sigma_{3}}, k \in L_{2}, \\
& \left(\chi(k, \xi) \mathrm{e}^{2 \mathrm{i} t \xi^{2}}(2 t)^{\frac{\mathrm{i} \nu}{2}}\right)^{\sigma_{3}}\left[\begin{array}{ccc}
1 & \frac{-r(\bar{k})}{1+r(k) \bar{r}(\bar{k})} \lambda^{-2 \mathrm{i} \nu} \mathrm{e}^{-2 \mathrm{i} \lambda^{2}} \\
0 & 1 & 1
\end{array}\right]\left(\chi(k, \xi) \mathrm{e}^{2 \mathrm{i} t \xi^{2}}(2 t)^{\frac{\mathrm{i} \nu}{2}}\right)^{-\sigma_{3}}, k \in L_{3}, \\
& \left(\chi(k, \xi) \mathrm{e}^{2 \mathrm{i} t \xi^{2}}(2 t)^{\frac{\mathrm{i} \nu}{2}}\right)^{\sigma_{3}}\left[\begin{array}{ccc}
\frac{-r(k)}{1+r(k) \bar{r}(\bar{k})} \lambda^{2 \mathrm{i} \nu} \mathrm{e}^{2 \mathrm{i} \lambda^{2}} & 1
\end{array}\right]\left(\chi(k, \xi) \mathrm{e}^{2 \mathrm{i} t \xi^{2}}(2 t)^{\frac{\mathrm{i} \nu}{2}}\right)^{-\sigma_{3}}, k \in L_{4},
\end{aligned}
$$




\subsubsection{Parabolic cylinder functions.}

The parabolic cylinder function $D_{a}(z)$ is an entire function, which satisfies the differential equation

$$
\partial_{z z} D_{a}(z)+\left(a+\frac{1}{2}-\frac{z^{2}}{4}\right) D_{a}(z)=0
$$

and has the asymptotics as $\lambda \rightarrow \infty$

$$
D_{a}(z)=z^{a} \mathrm{e}^{-z^{2} / 4}\left(1-\frac{a(a-1)}{2 z^{2}}+\frac{a(a-1)(a-2)(a-3)}{8 z^{4}}+\ldots\right), \quad \arg z \in\left(\frac{-3 \pi}{4}, \frac{3 \pi}{4}\right),
$$

or, in more general form,

$$
D_{a}(z)=z^{a} \mathrm{e}^{-z^{2} / 4}\left(\sum_{j=0}^{N-1} \frac{(-1)^{j} \cdot(a)_{(2 j)}}{j ! 2^{j} z^{2 j}}+\mathcal{O}\left(z^{-2 N}\right)\right), \quad \arg z \in\left(\frac{-3 \pi}{4}, \frac{3 \pi}{4}\right),
$$

where

$$
(a)_{(0)}=1, \quad(a)_{(n)}=a(a-1) \cdot \ldots \cdot(a-n+1)=\frac{a !}{(a-n) !}, \quad n \geq 1 .
$$

In particular case when $a=0$ we have

$$
D_{0}(z)=\mathrm{e}^{-z^{2} / 4}
$$

Furthermore, $D_{a}(z)$ satisfies the following relations:

$$
\begin{aligned}
& D_{a}(z)=\mathrm{e}^{-\pi a \mathrm{i}} D_{a}(-z)+\frac{\sqrt{2 \pi}}{\Gamma(-a)} \mathrm{e}^{-\pi(a+1) \mathrm{i} / 2} D_{-a-1}(\mathrm{i} z), \\
& D_{a}(z)=\mathrm{e}^{\pi a \mathrm{i}} D_{a}(-z)+\frac{\sqrt{2 \pi}}{\Gamma(-a)} \mathrm{e}^{\pi(a+1) \mathrm{i} / 2} D_{-a-1}(-\mathrm{i} z), \\
& D_{a}(z)=\frac{\Gamma(a+1)}{\sqrt{2 \pi}}\left(\mathrm{e}^{\pi \mathrm{i} a / 2} D_{-a-1}(\mathrm{i} z)+\mathrm{e}^{-\pi \mathrm{i} a / 2} D_{-a-1}(-\mathrm{i} z)\right), \\
& D_{a+1}(z)-z D_{a}(z)+a D_{a-1}(z)=0, \quad D_{a}^{\prime}(z)=-\frac{z}{2} D_{a}(z)+a D_{a-1}(z)=0 .
\end{aligned}
$$

Let us consider the following piece-wise analytic function:

$$
\begin{array}{cc}
\Psi(\lambda)=\left[\begin{array}{cc}
u^{+} D_{-\mathrm{i} \nu}\left(2 \mathrm{e}^{-3 \pi \mathrm{i} / 4} \lambda\right) & \beta_{1} v^{+} D_{\mathrm{i} \nu-1}\left(2 \mathrm{e}^{-\pi \mathrm{i} / 4} \lambda\right) \\
\beta_{2} u^{+} D_{-\mathrm{i} \nu-1}\left(2 \mathrm{e}^{-3 \pi \mathrm{i} / 4} \lambda\right) & v^{+} D_{\mathrm{i} \nu}\left(2 \mathrm{e}^{-\pi \mathrm{i} / 4} \lambda\right)
\end{array}\right], \quad \operatorname{Im} \lambda>0, \\
\Psi(\lambda)=\left[\begin{array}{cc}
u^{-} D_{-\mathrm{i} \nu}\left(2 \mathrm{e}^{\pi \mathrm{i} / 4} \lambda\right) & -\beta_{1} v^{-} D_{\mathrm{i} \nu-1}\left(2 \mathrm{e}^{3 \pi \mathrm{i} / 4} \lambda\right) \\
-\beta_{2} u^{-} D_{-\mathrm{i} \nu-1}\left(2 \mathrm{e}^{\pi \mathrm{i} / 4} \lambda\right) & v^{-} D_{\mathrm{i} \nu}\left(2 \mathrm{e}^{3 \pi \mathrm{i} / 4} \lambda\right)
\end{array}\right], \quad \operatorname{Im} \lambda<0,
\end{array}
$$

where

$$
\begin{aligned}
& u^{+}=2^{\mathrm{i} \nu} \mathrm{e}^{3 \pi \nu / 4}, \quad u^{-}=2^{\mathrm{i} \nu} \mathrm{e}^{-\pi \nu / 4}, \quad v^{+}=2^{-\mathrm{i} \nu} \mathrm{e}^{-\pi \nu / 4}, \quad v^{-}=2^{-\mathrm{i} \nu} \mathrm{e}^{3 \pi \nu / 4}, \\
& \beta_{1}=\frac{-\mathrm{i} \sqrt{2 \pi} 2^{2 \mathrm{i} \nu} \mathrm{e}^{\pi \nu / 2}}{r_{0} \Gamma(\mathrm{i} \nu)}=\frac{\rho_{0} \Gamma(-\mathrm{i} \nu+1) 2^{2 \mathrm{i} \nu}}{\sqrt{2 \pi} \mathrm{e}^{\pi \nu / 2}}, \quad \beta_{2}=\frac{r_{0} \Gamma(1+\mathrm{i} \nu)}{\sqrt{2 \pi} 2^{2 \mathrm{i} \nu} \mathrm{e}^{\pi \nu / 2}}=\frac{\mathrm{i} \sqrt{2 \pi} \mathrm{e}^{\pi \nu / 2}}{\rho_{0} 2^{2 \mathrm{i} \nu} \Gamma(-\mathrm{i} \nu)},
\end{aligned}
$$

and

$$
\nu=\frac{1}{2 \pi} \ln \left(1+r_{0} \rho_{0}\right),
$$

and $r_{0}, \rho_{0}$ are some (complex) parameters. Properties (5.10) allows to verify that $\Psi(\lambda)$ has the following jump across the real line:

$$
\Psi(\lambda-\mathrm{i} 0)=\Psi(\lambda+\mathrm{i} 0)\left(\begin{array}{cc}
1 & -\rho_{0} \\
-r_{0} & 1+r_{0} \rho_{0}
\end{array}\right), \quad \lambda \in \mathbb{R} .
$$


(Let us observe, that for $\nu=0$ the expression for $\Psi$ simplifies to $\Psi(\lambda)=\mathrm{e}^{-\mathrm{i} \lambda^{2} \sigma_{3}}, \beta_{1}=\beta_{2}=0$. The latter is due to the asymptotics $x \cdot \Gamma\left(\frac{\mathrm{i}}{2 \pi} \ln \left(1+x^{2}\right)\right)=\frac{-2 \mathrm{i} \pi}{x}(1+o(1)), x \rightarrow 0$. Furthermore, $\beta_{1}=\overline{\beta_{2}}$ if $\rho_{0}=\overline{r_{0}}$, and $\beta_{1}=-\overline{\beta_{2}}$ if $\left.\rho_{0}=-\overline{r_{0}}\right)$. Now let us take

$$
r_{0}=r\left(k_{0}\right), \quad \rho_{0}=\overline{r\left(k_{0}\right)} .
$$

The function

$$
\begin{aligned}
& P_{P C}(\lambda)=\Psi(\lambda) \cdot \lambda^{\mathrm{i} \nu \sigma_{3}} \mathrm{e}^{\mathrm{i} \lambda^{2} \sigma_{3}}, \arg \lambda \in\left(\frac{\pi}{4}, \frac{3 \pi}{4}\right) \cup\left(\frac{-3 \pi}{4}, \frac{-\pi}{4}\right), \\
&=\Psi(\lambda) \cdot \lambda^{\mathrm{i} \nu \sigma_{3}} \mathrm{e}^{\mathrm{i} \lambda^{2} \sigma_{3}}\left(\begin{array}{cc}
1 & 0 \\
-r\left(k_{0}\right) \lambda^{2 \mathrm{i} \nu} \mathrm{e}^{2 \mathrm{i} \lambda^{2}} & 1
\end{array}\right), \arg \lambda \in\left(0, \frac{\pi}{4}\right), \\
&=\Psi(\lambda) \cdot \lambda^{\mathrm{i} \nu \sigma_{3}} \mathrm{e}^{\mathrm{i} \lambda^{2} \sigma_{3}}\left(\begin{array}{ccc}
1 & \overline{r\left(k_{0}\right)} \lambda^{-2 \mathrm{i} \nu} \mathrm{e}^{-2 \mathrm{i} \lambda^{2}} \\
0 & 1
\end{array}\right), \arg \lambda \in\left(\frac{-\pi}{4}, 0\right), \\
&=\Psi(\lambda) \cdot \lambda^{\mathrm{i} \nu \sigma_{3}} \mathrm{e}^{\mathrm{i} \lambda^{2} \sigma_{3}}\left(\begin{array}{cc}
1 & \frac{-r\left(k_{0}\right)}{1+\left|r\left(k_{0}\right)\right|^{2}} \lambda^{-2 \mathrm{i} \nu} \mathrm{e}^{-2 \mathrm{i} \lambda^{2}} \\
0 & 1
\end{array}\right), \arg \lambda \in\left(\frac{3 \pi}{4}, \pi\right), \\
&=\Psi(\lambda) \cdot \lambda^{\mathrm{i} \nu \sigma_{3}} \mathrm{e}^{\mathrm{i} \lambda^{2} \sigma_{3}}\left(\begin{array}{cc}
\frac{r\left(k_{0}\right)}{1} & 0 \\
\frac{1+\left|r\left(k_{0}\right)\right|^{2}}{2 \mathrm{i} \nu} \mathrm{e}^{2 \mathrm{i} \lambda^{2}} & 1
\end{array}\right), \arg \lambda \in\left(-\pi,-\frac{3 \pi}{4}\right)
\end{aligned}
$$

satisfies the jump relations $P_{P C,-}(\lambda)=P_{P C,+}(\lambda) J_{P C}(\lambda)$ on the contour

$$
\begin{gathered}
\lambda \in \Sigma_{P C}=\left(\infty \mathrm{e}^{3 \pi \mathrm{i} / 4}, 0\right) \cup\left(\infty \mathrm{e}^{-3 \pi \mathrm{i} / 4}, 0\right) \cup\left(0, \infty \mathrm{e}^{\pi \mathrm{i} / 4}\right) \cup\left(0, \infty \mathrm{e}^{-\pi \mathrm{i} / 4}\right): \\
J_{P C}=\left[\begin{array}{cc}
1 & \frac{-\overline{r\left(k_{0}\right)}}{1+\left|r\left(k_{0}\right)\right|^{2}} \lambda^{-2 \mathrm{i} \nu} \mathrm{e}^{-2 \mathrm{i} \lambda^{2}} \\
0 & 1
\end{array}\right], \lambda \in\left(\infty \mathrm{e}^{3 \pi \mathrm{i} / 4}, 0\right), \quad=\left[\begin{array}{cc}
1 & 0 \\
-r\left(k_{0}\right) \lambda^{2 \mathrm{i} \nu} \mathrm{e}^{2 \mathrm{i} \lambda^{2}} & 1
\end{array}\right], \lambda \in\left(0, \infty \mathrm{e}^{\pi \mathrm{i} / 4}\right), \\
=\left[\begin{array}{cc}
1 & 0 \\
\frac{-r\left(k_{0}\right)}{1+\left|r\left(k_{0}\right)\right|^{2}} \lambda^{2 \mathrm{i} \nu} \mathrm{e}^{2 \mathrm{i} \lambda^{2}} & 1
\end{array}\right], \lambda \in\left(\infty \mathrm{e}^{-3 \pi \mathrm{i} / 4}, 0\right), \quad=\left[\begin{array}{cc}
1 & -\overline{r\left(k_{0}\right)} \lambda^{-2 \mathrm{i} \nu} \mathrm{e}^{-2 \mathrm{i} \lambda^{2}} \\
0 & 1
\end{array}\right], \lambda \in\left(0, \infty \mathrm{e}^{-\pi \mathrm{i} / 4}\right),
\end{gathered}
$$

and has the uniform asymptotics as $\lambda \rightarrow \infty$ of the following form:

$$
P_{P C}(\lambda)=\left[\begin{array}{cc}
1-\frac{\nu(1+\mathrm{i} \nu)}{8 \lambda^{2}}-\frac{\mathrm{i} \nu(1+\mathrm{i} \nu)(2+\mathrm{i} \nu)(3+\mathrm{i} \nu)}{128 \lambda^{4}}+\ldots & \frac{\mathrm{e}^{\pi \mathrm{i} / 4} \beta_{1}}{2 \lambda}+\frac{\mathrm{e}^{-\pi \mathrm{i} / 4} \beta_{1}(1-\mathrm{i} \nu)(2-\mathrm{i} \nu)}{16 \lambda^{3}}+\ldots \\
\frac{\mathrm{e}^{3 \pi \mathrm{i} / 4} \beta_{2}}{2 \lambda}+\frac{\mathrm{e}^{-3 \pi \mathrm{i} / 4} \beta_{2}(1+\mathrm{i} \nu)(2+\mathrm{i} \nu)}{16 \lambda^{3}}+\ldots & 1-\frac{\nu(1-\mathrm{i} \nu)}{8 \lambda^{2}}+\frac{\mathrm{i} \nu(1-\mathrm{i} \nu)(2-\mathrm{i} \nu)(3-\mathrm{i} \nu)}{128 \lambda^{4}}+\ldots
\end{array}\right] .
$$

\subsection{First approximation of $M^{(1)}$.}

We look for an approximation of $M^{(3)}$ of the form

$$
M_{\infty}=\left\{\begin{array}{l}
\left(\frac{k-E_{0}}{k-\bar{E}_{0}}\right)^{-n \sigma_{3}}, \quad\left|k-E_{0}\right|>r,\left|k-\bar{E}_{0}\right|>r,\left|k-k_{0}\right|>\delta, \\
B_{u} L(-\phi(k, \xi))^{\sigma_{3} / 2} t^{\gamma \sigma_{3}} \mathrm{e}^{-\mathrm{i} \varphi(t) \sigma_{3}}, \quad\left|k-E_{0}\right|<r \\
B_{d} L_{d}(-\bar{\phi}(\bar{k}, \xi))^{-\sigma_{3} / 2} t^{-\gamma \sigma_{3}} \mathrm{e}^{-\mathrm{i} \varphi(t) \sigma_{3}}, \quad\left|k-\bar{E}_{0}\right|<r, \\
B_{P C}(k) P_{P C}(\lambda) \cdot\left(\chi(k, \xi) \mathrm{e}^{2 \mathrm{i} t \xi^{2}}(2 t)^{\mathrm{i} \nu}\right)^{-\sigma_{3}},\left|k-k_{0}\right|<\delta .
\end{array}\right.
$$

In the process of the construction we will also determine the matrix-valued functions $B_{u}, B_{d}, B_{P C}$ analytic inside the disks $\left|k-E_{0}\right|<r,\left|k-\bar{E}_{0}\right|<r,\left|k-k_{0}\right|<\delta$, respectively. The driving logic is 
that of minimizing the distance of the error matrix $E=M^{(3)} M_{\infty}^{-1}$ from the identity matrix. To this end we inspect its jump $J_{E}=\left(E_{+}\right)^{-1} E_{-}=M_{\infty,+} J^{(3)} M_{\infty,-}^{-1}$.

On the interval $l^{+}$the jump is

$$
J_{E}=B_{u} L_{+}(-\phi)^{\sigma_{3} / 2} t^{\gamma \sigma_{3}} \mathrm{e}^{-\mathrm{i} \varphi(t) \sigma_{3}}\left(\begin{array}{cc}
1 & 0 \\
\phi t^{2 \gamma} \mathrm{e}^{-2 \mathrm{i} \varphi(t)} \sqrt{\zeta} \mathrm{e}^{-\zeta} & 1
\end{array}\right) \mathrm{e}^{\mathrm{i} \varphi(t) \sigma_{3}} t^{-\gamma \sigma_{3}}(-\phi)^{-\sigma_{3} / 2} L_{-}^{-1} B_{u}^{-1}=I .
$$

Similarly, on $l^{-}$the jump $J_{E}=I$. Furthermore, on the parts $L_{j}, j=1, \ldots, 4$, of the contour within the disk $D=\left\{k:\left|k-k_{0}\right|<\delta\right\}$ the jump is

$$
J_{E}=B_{P C} \cdot P_{P C,+} \cdot\left(\chi(k, \xi) \mathrm{e}^{2 \mathrm{i} t \xi^{2}}(2 t)^{\frac{\mathrm{i} \nu}{2}}\right)^{-\sigma_{3}} \cdot J^{(3)} \cdot\left(\chi(k, \xi) \mathrm{e}^{2 \mathrm{i} t \xi^{2}}(2 t)^{\frac{\mathrm{i} \nu}{2}}\right)^{\sigma_{3}} \cdot J_{P C}^{-1} \cdot P_{P C,+}^{-1} B_{P C}^{-1}
$$

Since $r(k)$ is continuous in the neighborhood of the point $k=k_{0}$, the terms

$$
\left(\frac{r(k) \chi\left(k_{0}\right)}{\chi(k)}-r\left(k_{0}\right)\right) \lambda^{2 \mathrm{i} \nu} \mathrm{e}^{2 \mathrm{i} \lambda^{2}}, \quad\left(\frac{r(k) \chi\left(k_{0}\right)}{\chi(k)(1+r(k) \bar{r}(\bar{k}))}-\frac{r\left(k_{0}\right)}{1+r\left(k_{0}\right)^{2}}\right) \lambda^{2 \mathrm{i} \nu} \mathrm{e}^{2 \mathrm{i} \lambda^{2}},
$$

which appear in the jump on the parts of the segments $L_{1}, L_{4}$, can be estimated as

$$
\mathcal{O}\left(\left|k-k_{0}\right|\right) \cdot \mathrm{e}^{-2|\lambda|^{2}}=\mathcal{O}(|\mu|) \cdot \mathrm{e}^{-4 t|\mu|^{2}}=t^{-\frac{1}{2}} \cdot \mathcal{O}(|\mu| \sqrt{t}) \cdot \mathrm{e}^{-4 t|\mu|^{2}}=\mathcal{O}\left(t^{-1 / 2}\right) .
$$

The ingredients on the parts of the segments $L_{2}, L_{3}$ admit similar estimate. Hence, the jump $J_{E}$ is estimated as

$$
J_{E}=\mathbf{1}+\mathcal{O}\left(t^{-1 / 2}\right), \quad k \in\left(L_{1} \cup L_{2} \cup L_{3} \cup L_{4}\right) \cap D,
$$

provided that $B_{P C}$ is bounded in $D$ uniformly w.r.t $t$. Similarly, it follows from (5.11) that for $k \in \partial D:\left|k-k_{0}\right|=\delta$,

$$
J_{E}=I+\mathcal{O}\left(t^{-1 / 2}\right), \quad k \in \partial D .
$$

Furthermore, the jump $J_{E}$ on the disks

$$
\partial C=\left\{k:\left|k-E_{0}\right|=r\right\}, \quad \partial C_{d}=\left\{k:\left|k-\bar{E}_{0}\right|=r\right\}, \quad \partial D=\left\{k:\left|k-k_{0}\right|=\delta\right\}
$$

(we take counterclockwise orientation) is

$$
\begin{aligned}
& J_{E}=B_{u} L(-\phi(k, \xi))^{\sigma_{3} / 2} t^{\gamma \sigma_{3}} \mathrm{e}^{-\mathrm{i} \varphi(t) \sigma_{3}}\left(\frac{k-E_{0}}{k-\bar{E}_{0}}\right)^{n \sigma_{3}}, k \in \partial C, \\
& J_{E}=B_{d} L_{d}(-\bar{\phi}(\bar{k}, \xi))^{-\sigma_{3} / 2} t^{-\gamma \sigma_{3}} \mathrm{e}^{-\mathrm{i} \varphi(t) \sigma_{3}}\left(\frac{k-E_{0}}{k-\bar{E}_{0}}\right)^{n \sigma_{3}}, k \in \partial C_{d}, \\
& J_{E}=B_{P C}(k) P_{P C}(\lambda) \cdot\left(\chi\left(k_{0}, \xi\right) \mathrm{e}^{2 \mathrm{i} t \xi^{2}}(2 t)^{\mathrm{i} \nu / 2}\right)^{-\sigma_{3}}\left(\frac{k-E_{0}}{k-\bar{E}_{0}}\right)^{n \sigma_{3}}, k \in \partial D .
\end{aligned}
$$

To have $J_{E}$ close to $I$, taking into account the asymptotics (5.4), (5.6), (5.11) of $L, L_{d}, P_{P C}$ on the circles (we have $\zeta=z t \rightarrow \infty$ as $t \rightarrow \infty$ when $k \in \partial C$ and $\lambda=\sqrt{2 t} \mu \rightarrow \infty$ for $k \in \partial D$ ), we take

$$
\begin{aligned}
& B_{u}=\left(\frac{k-E_{0}}{k-\bar{E}_{0}} \cdot \frac{1}{z}\right)^{-n \sigma_{3}}\left(\sqrt{-\phi} t^{(\gamma-n)} \mathrm{e}^{-\mathrm{i} \varphi(t)}\right)^{-\sigma_{3}}, \quad B_{d}=\left(\frac{k-E_{0}}{k-\bar{E}_{0}} \cdot z_{d}\right)^{-n \sigma_{3}}\left(\sqrt{-\bar{\phi}(\bar{k})} t^{\gamma-n} \mathrm{e}^{\mathrm{i} \varphi(t)}\right)^{\sigma_{3}}, \\
& B_{P C}(k)=\left(\chi\left(k_{0}, \xi\right) \mathrm{e}^{2 \mathrm{i} t \xi^{2}}(2 t)^{\frac{\mathrm{i} \nu}{2}}\right)^{\sigma_{3}} \cdot\left(\frac{k-E_{0}}{k-\bar{E}_{0}}\right)^{-n \sigma_{3}} .
\end{aligned}
$$


We see that indeed $B_{u}, B_{d}$ do not have poles at $k=E_{0}, k=\overline{E_{0}}$, i.e. at $z=0, z_{d}=0$, and that $B_{u}, B_{d}, B_{P C}$ are indeed bounded inside the circles uniformly w.r.t. $t$. Furthermore,

$$
\begin{aligned}
& J_{E}=\left(\frac{k-\bar{E}_{0}}{k-E_{0}} \cdot z\right)^{n \sigma_{3}}(-\phi(k, \xi))^{\frac{-\sigma_{3}}{2}} t^{(n-\gamma) \sigma_{3}} \mathrm{e}^{\mathrm{i} \varphi(t) \sigma_{3}}\left(\begin{array}{cc}
1+\mathcal{O}\left(\frac{1}{z t}\right) & \mathcal{O}\left(\frac{1}{z t}\right) \\
\mathcal{O}\left(\frac{1}{z t}\right) & 1+\mathcal{O}\left(\frac{1}{z t}\right)
\end{array}\right) \\
&(-\phi(k, \xi))^{\frac{\sigma_{3}}{2}} t^{(\gamma-n) \sigma_{3}} \mathrm{e}^{-\mathrm{i} \varphi(t) \sigma_{3}}\left(\frac{k-E_{0}}{k-\bar{E}_{0}} \cdot \frac{1}{z}\right)^{n \sigma_{3}}, k \in \partial C \\
& J_{E}=\left(\frac{k-\bar{E}_{0}}{k-E_{0}} \cdot \frac{1}{z_{d}}\right)^{n \sigma_{3}}(-\bar{\phi}(\bar{k}, \xi))^{\frac{\sigma_{3}}{2}} t^{(\gamma-n) \sigma_{3}} \mathrm{e}^{\mathrm{i} \varphi(t) \sigma_{3}}\left(\begin{array}{cc}
1+\mathcal{O}\left(\frac{1}{z_{d} t}\right) & \mathcal{O}\left(\frac{1}{z_{d} t}\right) \\
\mathcal{O}\left(\frac{1}{z_{d} t}\right) & 1+\mathcal{O}\left(\frac{1}{z_{d} t}\right)
\end{array}\right) . \\
&(-\bar{\phi}(\bar{k}, \xi))^{\frac{-\sigma_{3}}{2}} t^{(n-\gamma) \sigma_{3}} \mathrm{e}^{-\mathrm{i} \varphi(t) \sigma_{3}}\left(\frac{k-E_{0}}{k-\bar{E}_{0}} \cdot z_{d}\right)^{n \sigma_{3}}, k \in \partial C_{d}, \\
& J_{E}=\left(\chi\left(k_{0}, \xi\right) \mathrm{e}^{2 \mathrm{i} t \xi^{2}}(2 t)^{\frac{\mathrm{i} \nu}{2}}\right)^{\sigma_{3}} \cdot\left(\frac{k-E_{0}}{k-\bar{E}_{0}}\right)^{-n \sigma_{3}}\left(\begin{array}{cc}
1+\mathcal{O}\left(\frac{1}{\mu^{2} t}\right) & \mathcal{O}\left(\frac{1}{\mu \sqrt{t}}\right) \\
\mathcal{O}\left(\frac{1}{\mu \sqrt{t}}\right) & 1+\mathcal{O}\left(\frac{1}{\mu^{2} t}\right)
\end{array}\right) . \\
&\left(\chi\left(k_{0}, \xi\right) \mathrm{e}^{2 \mathrm{i} t \xi^{2}}(2 t)^{\frac{\mathrm{i} \nu}{2}}\right)^{-\sigma_{3}} \cdot\left(\frac{k-E_{0}}{k-\bar{E}_{0}}\right)^{n \sigma_{3}}, k \in \partial D,
\end{aligned}
$$

and hence

$$
\begin{aligned}
& J_{E}=\left(\begin{array}{cc}
1+\mathrm{O}\left(t^{-1}\right) & \mathrm{O}\left(t^{-2 \gamma+2 n-1}\right) \\
\mathrm{O}\left(t^{2 \gamma-2 n-1}\right) & 1+\mathrm{O}\left(t^{-1}\right)
\end{array}\right), k \in \partial C, \quad J_{E}=\left(\begin{array}{cc}
1+\mathrm{O}\left(t^{-1}\right) & \mathrm{O}\left(t^{2 \gamma-2 n-1}\right) \\
\mathrm{O}\left(t^{-2 \gamma+2 n-1}\right) & 1+\mathrm{O}\left(t^{-1}\right)
\end{array}\right), k \in \partial C_{d} . \\
& J_{E}=\left(\begin{array}{cc}
1+\mathrm{O}\left(t^{-1}\right) & \mathrm{O}\left(t^{-1 / 2}\right) \\
\mathrm{O}\left(t^{-1 / 2}\right) & 1+\mathrm{O}\left(t^{-1}\right)
\end{array}\right), k \in \partial D .
\end{aligned}
$$

For every value of $\gamma$ which is not half-integer we can choose $n$ such that $\gamma-\frac{1}{2}<n<\gamma+\frac{1}{2}$, and both off-diagonal terms in the r.h.s. of the first expression in (5.17) will be vanishing. However, for a half-integer $\gamma=m+\frac{1}{2}$ we cannot make both off-diagonal terms in (5.17) small: indeed, if we choose $n=m$, then the $(1,2)$ entry is of the order $\mathcal{O}\left(t^{-2}\right)$, but the $(2,1)$ entry is just $\mathcal{O}(1)$; vice versa, for $n=m+1$, then $(2,1)$ entry is $\mathcal{O}\left(t^{-2}\right)$, but the $(1,2)$ entry is just $\mathcal{O}(1)$. As we shall see below, this indicates the presence of asymptotic solitons, which correspond to half-integer $\gamma$. Away from the asymptotic solitons, the solution of NLS equation is asymptotically vanishing.

To capture the asymptotic solitons, we are lead to make a further correction in the approximate solution; this is accomplished in the next section.

\subsubsection{Refined approximation of $M^{(3)}$}

In order to have more freedom in choosing $n$ in (5.17), the idea is that of "removing" the $\frac{1}{\zeta}$ term in $(2,1)$ entry in asymptotics $(5.7)$ of $L$, and in $(1,2)$ entry in asymptotics $(5.8)$ of $L_{d}$ at $\infty$, so that they will start from $\zeta^{-2}, \zeta_{d}^{-2}$,

$$
\left(\begin{array}{cc}
1 & 0 \\
\frac{R_{1}}{\zeta} & 1
\end{array}\right) L=\left(\begin{array}{cc}
1+\mathcal{O}\left(\frac{1}{\zeta}\right) & \mathcal{O}\left(\frac{1}{\zeta}\right) \\
\mathcal{O}\left(\frac{1}{\zeta^{2}}\right) & 1+\mathcal{O}\left(\frac{1}{\zeta}\right)
\end{array}\right), \quad\left(\begin{array}{cc}
1 & \frac{R_{1}}{\zeta_{d}} \\
0 & 1
\end{array}\right) L_{d}=\left(\begin{array}{cc}
1+\mathcal{O}\left(\frac{1}{\zeta_{d}}\right) & \mathcal{O}\left(\frac{1}{\zeta_{d}^{2}}\right) \\
\mathcal{O}\left(\frac{1}{\zeta_{d}}\right) & 1+\mathcal{O}\left(\frac{1}{\zeta_{d}}\right)
\end{array}\right)
$$

where

$$
R_{1}=\frac{n ! \Gamma\left(n+\frac{3}{2}\right)}{2 \pi \mathrm{i}} .
$$

However, this will bring poles at $k=E_{0}, k=\bar{E}_{0}$ of the approximate solution and to compensate for this issue we multiply all $M_{\infty}$ by an appropriate meromorphic matrix function from the left, 
which will remove these poles. In concrete, the above idea requires to define

$$
\begin{gathered}
G=I+\frac{\mathcal{A}}{k-E_{0}}+\frac{\widetilde{\mathcal{A}}}{k-\bar{E}_{0}} \\
M_{\infty}^{(1)}=\left\{\begin{array}{l}
G\left(\frac{k-E_{0}}{k-\bar{E}_{0}}\right)^{-n \sigma_{3}}, \quad\left|k-E_{0}\right|>r,\left|k-\bar{E}_{0}\right|>r,\left|k-k_{0}\right|>\delta, \\
G B_{u}\left(\begin{array}{cc}
1 & 0 \\
\frac{R_{1}}{\zeta} & 1
\end{array}\right) L(-\mathrm{i} \phi)^{\sigma_{3} / 2} t^{\gamma \sigma_{3}}, \quad|k-\mathrm{i} c|<r, \\
G B_{d}\left(\begin{array}{cc}
1 & \frac{R_{1}}{\zeta_{d}} \\
0 & 1
\end{array}\right) L_{d}\left(\mathrm{i} \phi_{d}\right)^{-\sigma_{3} / 2} t^{-\gamma \sigma_{3}}, \quad|k+\mathrm{i} c|<r, \\
G B_{P C}(k) P_{P C}(\lambda) \cdot\left(\chi(k, \xi) \mathrm{e}^{2 \mathrm{i} t \xi^{2}}(2 t)^{\frac{\mathrm{i} \nu}{2}}\right)^{-\sigma_{3}},\left|k-k_{0}\right|<\delta,
\end{array}\right.
\end{gathered}
$$

where $B_{u}, B_{d}, B_{P C}$ are as in (5.13). The new error matrix

$$
E^{(1)}:=M^{(3)}\left(M_{\infty}^{(1)}\right)^{-1}
$$

has the jump

$$
J_{E^{(1)}}=\left(E_{+}^{(1)}\right)^{-1} E_{-}^{(1)}=M_{\infty,+}^{(1)} J^{(3)}\left(M_{\infty,-}^{(1)}\right)^{-1} ;
$$

in the intervals $l^{ \pm}$the jump is $J_{E^{(1)}}=I$, on $k \in(\Sigma \cap D) \cup \partial D$ the jump is $J_{E^{(1)}}=\mathbf{1}+\mathcal{O}\left(t^{-1 / 2}\right)$, and on the circles $k \in \partial C, k \in \partial C_{d}$ it is of the form

$$
\begin{aligned}
& J_{E^{(1)}}=G\left(\begin{array}{cc}
1+\mathrm{O}\left(t^{-1}\right) & \mathrm{O}\left(t^{-2 \gamma+2 n-1}\right) \\
\mathrm{O}\left(t^{2 \gamma-2 n-2}\right) & 1+\mathrm{O}\left(t^{-1}\right)
\end{array}\right) G^{-1}, k \in \partial C \\
& J_{E^{(1)}}=G\left(\begin{array}{cc}
1+\mathrm{O}\left(t^{-1}\right) & \mathrm{O}\left(t^{2 \gamma-2 n-2}\right) \\
\mathrm{O}\left(t^{-2 \gamma+2 n-1}\right) & 1+\mathrm{O}\left(t^{-1}\right)
\end{array}\right) G^{-1}, k \in \partial C_{d} .
\end{aligned}
$$

We see that (5.20) provides us with better estimate than (5.17) provided that $G, G^{-1}$ are uniformly bounded, as $t \rightarrow \infty$, on the circles $|k \mp \mathrm{i} c|=r$. Now, for such $\gamma$ that

$$
\{\gamma\} \in\left[0, \frac{1}{2}\right]
$$

we choose

$$
n=\lfloor\gamma\rfloor,
$$

where $\{\gamma\},\lfloor\gamma\rfloor$ denote the fractional part of $\gamma$ and the greatest integer not exceeding $\gamma$, respectively. Then $J_{E^{(1)}}$ in (5.20) admits the estimate

$$
J_{E^{(1)}}=I+\mathcal{O}\left(t^{-1}\right)
$$

Other values of $\gamma$, such that

$$
\{\gamma\} \in\left(\frac{1}{2}, 1\right)
$$

are considered in the next section 5.2.3.

We determine now the matrix $G(5.18)$ in such a way that $M_{\infty}^{(1)}$ is bounded (has no poles) at $k=E_{0}, k=\bar{E}_{0}$. Expanding the product, the terms responsible for poles at $k=E_{0}, k=\bar{E}_{0}$ in $M_{\infty}^{(1)}$ are

$$
G B_{u}\left(\begin{array}{cc}
1 & 0 \\
\frac{R_{1}}{\zeta} & 1
\end{array}\right)=\left(I+\frac{\mathcal{A}}{k-E_{0}}+\frac{\widetilde{\mathcal{A}}}{k-\bar{E}_{0}}\right)\left(\begin{array}{cc}
1 & 0 \\
\frac{R_{1}}{z} z^{-2 n}\left(\frac{k-E_{0}}{k-\bar{E}_{0}}\right)^{2 n} t^{2 \gamma-2 n-1} \mathrm{e}^{-2 \mathrm{i} \varphi(t)}(-\phi) & 1
\end{array}\right) B_{u}
$$




$$
G B_{d}\left(\begin{array}{cc}
1 & \frac{R_{1}}{\zeta_{d}} \\
0 & 1
\end{array}\right)=\left(I+\frac{\mathcal{A}}{k-E_{0}}+\frac{\widetilde{\mathcal{A}}}{k-\bar{E}_{0}}\right)\left(\begin{array}{cc}
1 & \frac{R_{1}}{z_{d}} z_{d}^{-2 n}\left(\frac{k-E_{0}}{k-\bar{E}_{0}}\right)^{-2 n}(-\bar{\phi}(\bar{k})) t^{2 \gamma-2 n-1} \mathrm{e}^{2 \mathrm{i} \varphi(t)} \\
0 & 1
\end{array}\right) B_{d} .
$$

We see that at most we can have the poles of the second order at $z=0, z_{d}=0$. The requirement that the singular part vanishes yields a linear system for the matrices $A, \widetilde{A}$ : from the vanishing of the double-pole coefficient it is seen that they must be of the form

$$
\mathcal{A}=\left(\begin{array}{ll}
a_{1} & 0 \\
b_{1} & 0
\end{array}\right), \quad \widetilde{\mathcal{A}}=\left(\begin{array}{cc}
0 & \widetilde{b}_{1} \\
0 & \widetilde{a}_{1}
\end{array}\right) .
$$

Writing down the conditions of vanishing of the residue we get the system of equations

$$
\left\{\begin{array} { l } 
{ \widetilde { b } _ { 1 } + ( 1 - \frac { a _ { 1 } } { 2 \mathrm { i } B } ) \frac { H _ { d } } { z _ { d } ^ { \prime } } = 0 , } \\
{ \widetilde { a } _ { 1 } - \frac { b _ { 1 } } { 2 \mathrm { i } B } \cdot \frac { H _ { d } } { z _ { d } ^ { \prime } } = 0 ; }
\end{array} \quad \left\{\begin{array}{l}
b_{1}+\left(1+\frac{\widetilde{a}_{1}}{2 \mathrm{i} B}\right) \frac{H}{z^{\prime}}=0 \\
a_{1}+\frac{\widetilde{b}_{1}}{2 \mathrm{i} B} \cdot \frac{H}{z^{\prime}}=0
\end{array}\right.\right.
$$

which decomposes into 2 linear systems: one for $a_{1}, \widetilde{b}_{1}$, another for $\widetilde{a}_{1}, b_{1}$. Here

$$
z^{\prime}:=8 B+\frac{2 \mathrm{i} \rho \ln t}{B t}, \quad z_{d}^{\prime}:=8 B-\frac{2 \mathrm{i} \rho \ln t}{B t},
$$

are the derivatives of $z, z_{d}$ w.r.t. $y, y_{d}$ respectively at the points $y=0, y_{d}=0$, and

$$
\begin{aligned}
& H=R_{1} t^{2 \gamma-2 n-1} \mathrm{e}^{-2 \mathrm{i} \varphi(t)} \lim _{k \rightarrow E_{0}}\left(\frac{k-E_{0}}{z\left(k-\bar{E}_{0}\right)}\right)^{2 n}(-\phi(k, \xi))=\frac{-R_{1} t^{2 \gamma-2 n-1}}{\mathrm{e}^{2 \mathrm{i} \varphi(t)}}\left(\frac{1}{2 \mathrm{i} B z^{\prime}}\right)^{2 n} \frac{\hat{\phi}\left(E_{0}\right)}{\delta^{2}\left(E_{0}, \xi\right) \sqrt{z^{\prime}}}= \\
& =\frac{(-1)^{n+1} n ! \Gamma\left(n+\frac{3}{2}\right) t^{2 \gamma-2 n-1} \mathrm{e}^{-2 \mathrm{i} \varphi(t)} \sqrt{2 B} \hat{\phi}\left(E_{0}\right)}{2 \pi \mathrm{i} \delta^{2}\left(E_{0}, \xi\right)\left[2 B\left(8 B+\frac{2 \mathrm{i} \rho \ln t}{B t}\right)\right]^{2 n+\frac{1}{2}}}, \\
& H_{d}=R_{1} t^{2 \gamma-2 n-1} \mathrm{e}^{2 \mathrm{i} \varphi(t)} \lim _{k \rightarrow \bar{E}_{0}}\left(\frac{z_{d}\left(k-E_{0}\right)}{k-\bar{E}_{0}}\right)^{-2 n}(-\bar{\phi}(\bar{k}, \xi))=\frac{-R_{1} t^{2 \gamma-2 n-1} \mathrm{e}^{2 \mathrm{i} \varphi(t)}}{\left(-2 \mathrm{i} B z_{d}\right)^{2 n}} \cdot \frac{\overline{\hat{\phi}\left(E_{0}\right)}}{{\overline{\delta\left(E_{0}, \xi\right)}}^{2}{\sqrt{z_{d}^{\prime}}}^{2}}= \\
& =\frac{(-1)^{n+1} n ! \Gamma\left(n+\frac{3}{2}\right) t^{2 \gamma-2 n-1} \mathrm{e}^{2 \mathrm{i} \varphi(t)} \sqrt{2 B} \overline{\hat{\phi}\left(E_{0}\right)}}{2 \pi \mathrm{i}{\overline{\delta\left(E_{0}, \xi\right)}}^{2}\left[2 B\left(8 B-\frac{2 \mathrm{i} \rho \ln t}{B t}\right)\right]^{2 n+\frac{1}{2}}},
\end{aligned}
$$

We have the symmetry

$$
\bar{H}_{d}=-H .
$$

Solving system (5.21) for $a_{1}, b_{1}, \widetilde{a}_{1}, \widetilde{b}_{1}$, we obtain

$$
\begin{aligned}
& a_{1}=\frac{-2 \mathrm{i} B H H_{d}}{4 B^{2} z^{\prime} z_{d}^{\prime}-H H_{d}}=2 \mathrm{i} B \frac{\left|\frac{H}{2 B z^{\prime}}\right|}{\left|\frac{2 B z^{\prime}}{H}\right|+\left|\frac{H}{2 B z^{\prime}}\right|}, \quad b_{1}=\frac{-4 B^{2} H z_{d}^{\prime}}{4 B^{2} z^{\prime} z_{d}^{\prime}-H H_{d}}=-\overline{\widetilde{b}_{1}}, \\
& \widetilde{a}_{1}=\frac{2 \mathrm{i} B H H_{d}}{4 B^{2} z^{\prime} z_{d}^{\prime}-H H_{d}}=-a_{1}, \quad \widetilde{b}_{1}=\frac{-4 B^{2} H_{d} z^{\prime}}{4 B^{2} z^{\prime} z_{d}^{\prime}-H H_{d}}=-2 B \frac{\mathrm{e}^{-\mathrm{i} \arg \left(\frac{H}{2 B z^{\prime}}\right)}}{\left|\frac{2 B z^{\prime}}{H}\right|+\left|\frac{H}{2 B z^{\prime}}\right|} .
\end{aligned}
$$

We see, that $a_{1}, b_{1}, \widetilde{a}_{1}, \widetilde{b}_{1}$ are all bounded for $t \rightarrow \infty$, hence, $G$ does not contribute to the error estimate (5.20) of $J_{E}$. Hence,

$$
q_{\infty}^{(1)}(x, t):=2 \mathrm{i} \lim _{k \rightarrow \infty} k\left(M_{\infty}\right)_{12}=-2 \mathrm{i} \lim _{k \rightarrow \infty} \overline{k\left(M_{\infty}\right)_{21}}=2 \mathrm{i} \widetilde{b}_{1}=-2 \mathrm{i} \overline{b_{1}}=\frac{2 \mathrm{i} B \cdot \frac{\bar{H}}{|H|} \cdot \frac{z^{\prime}}{\left|z^{\prime}\right|}}{\frac{1}{2}\left(\frac{2 B\left|z^{\prime}\right|}{|H|}+\frac{|H|}{2 B\left|z^{\prime}\right|}\right)} .
$$


Let us notice that

$$
\frac{\bar{H}}{|H|}=\mathrm{i}(-1)^{n+1} \mathrm{e}^{2 \mathrm{i} \varphi(t)-\mathrm{i} \arg \hat{\phi}\left(E_{0}\right)+2 \mathrm{i} \arg \delta\left(E_{0}\right)+\mathrm{i}\left(2 n+\frac{1}{2}\right) \operatorname{arctg} \frac{\rho \ln t}{4 B^{2} t}}, \quad \frac{z^{\prime}}{\left|z^{\prime}\right|}=\mathrm{e}^{\mathrm{i} \operatorname{arctg} \frac{\rho \ln t}{4 B^{2} t}},
$$

and

$$
\frac{|H|}{2 B\left|z^{\prime}\right|}=\frac{n ! \Gamma\left(n+\frac{3}{2}\right)}{2 \pi} \cdot \frac{\exp \left(-2 B(x+4 A t)-\left(2 n+\frac{3}{2}\right) \ln t\right) \cdot\left|\hat{\phi}\left(E_{0}\right)\right| \sqrt{2 B}}{\left|2 B\left(8 B+\frac{2 \mathrm{i} \rho \ln t}{B t}\right)\right|^{2 n+\frac{3}{2}}\left|\delta^{2}\left(E_{0}, \xi\right)\right|}
$$

Hence

$$
q_{\infty}^{(1)}=\frac{(-1)^{n} 2 B \exp \left[2 \mathrm{i} \varphi(t)-\mathrm{i} \arg \hat{\phi}\left(E_{0}\right)+2 \mathrm{i} \arg \delta\left(E_{0}, \xi\right)\right]}{\cosh \left[2 B(x+4 A t)+\left(2 n+\frac{3}{2}\right) \ln t+\ln \left(\frac{2 \pi}{n ! \Gamma\left(n+\frac{3}{2}\right)} \cdot \frac{\left|\delta\left(E_{0}\right)\right|^{2}\left(16 B^{2}\right)^{2 n+\frac{3}{2}}}{\left|\hat{\phi}\left(E_{0}\right)\right| \sqrt{2 B}}\right)\right]}+\mathcal{O}\left(\frac{\ln t}{t}\right)
$$

where

$$
\varphi(t)=2 t\left(A^{2}+B^{2}\right)+\frac{A \rho \ln t}{B}=-\left(A x+2 t\left(A^{2}-B^{2}\right)\right) .
$$

Let us observe for future reference, that formulas (5.22), (5.23) imply

$$
a_{1}-\mathrm{i} B=i B \frac{\left|\frac{H}{2 B z^{\prime}}\right|-\left|\frac{2 B z^{\prime}}{H}\right|}{\left|\frac{H}{2 B z^{\prime}}\right|+\left|\frac{2 B z^{\prime}}{H}\right|}, \quad \tilde{b}_{1}=-2 B \frac{\mathrm{e}^{-\mathrm{i} \arg \left(\frac{H}{2 B z^{\prime}}\right)}}{\left|\frac{H}{2 B z^{\prime}}\right|+\left|\frac{2 B z^{\prime}}{H}\right|},
$$

and since $\tilde{b}_{1}=\frac{q_{\infty}^{(1)}}{2 \mathrm{i}}$, then

$$
\left(a_{1}-\mathrm{i} B\right)^{2}=-B^{2}\left(1-\frac{\left|q_{\infty}^{(1)}\right|^{2}}{4 B^{2}}\right), \quad\left(\tilde{b}_{1}\right)^{2}=\frac{-\left(q_{\infty}^{(1)}\right)^{2}}{4} .
$$

Furthermore, the solution of the initial value problem $q(x, t)$ is equal to

$$
q(x, t)=q_{\infty}^{(1)}+q_{\text {err }}^{(1)}, \quad \text { where } \quad q_{\text {err }}^{(1)}:=2 \mathrm{i} \lim _{k \rightarrow \infty} k\left(E^{(1)}\right) 12,
$$

and it follows from $J_{E^{(1)}}=I+\mathcal{O}\left(t^{-1 / 2}\right)$ that

$$
q_{e r r}^{(1)}=\mathcal{O}\left(t^{-1 / 2}\right)
$$

and hence

$$
q(x, t)=q_{\infty}^{(1)}(x, t)+\mathcal{O}\left(t^{-1 / 2}\right) .
$$

However, in the next subsection we evaluate the error term $q_{e r r}^{(1)}$ more precisely, with accuracy up to $t^{-1}$.

\subsubsection{Calculation of $q_{e r r}^{(1)}$.}

Denote by $\Sigma_{E}$ the jump contour for the error matrix $E^{(1)}$. The function $E^{(1)}$ admits the representation

$$
E^{(1)}=\mathbf{1}+\mathcal{C}\left[E_{+}^{(1)} \cdot\left(\mathbf{1}-J_{E^{(1)}}\right)\right],
$$

where $\mathcal{C}$ is the Cauchy operator

$$
\mathcal{C} f=\frac{1}{2 \pi \mathrm{i}} \int_{\Sigma_{E}} \frac{f(s) \mathrm{d} s}{s-k}
$$


and $E_{+}^{(1)}$ is the solution of the small-norm singular integral equation

$$
E_{+}^{(1)}=\mathbf{1}+\mathcal{C}_{+}\left[E_{+}^{(1)} \cdot\left(\mathbf{1}-J_{E^{(1)}}\right)\right] .
$$

Developing the representation for $E^{(1)}$ into terms small w.r.t. $t$, we obtain

$$
E^{(1)}=\mathbf{1}+\mathcal{C}\left[\mathbf{1}-J_{E^{(1)}}\right]+\mathcal{C}\left[\left(E_{+}^{(1)}-\mathbf{1}\right) \cdot\left(\mathbf{1}-J_{E^{(1)}}\right)\right],
$$

and the last summand in the latter formula is already of the order $\mathcal{O}\left(t^{-1}\right)$, because of presence of product of two terms of the order $t^{-1 / 2}$, namely

$$
E_{+}^{(1)}-\mathbf{1}=\mathcal{O}\left(t^{-1 / 2}\right) \quad \text { and } \quad J_{E^{(1)}}-\mathbf{1}=\mathcal{O}\left(t^{-1 / 2}\right) .
$$

Furthermore, the second summand in (5.26) involves integration over the circles $\partial C, \partial C_{d}, \partial D$, segments $\Sigma_{E} \cap D$, and other lenses, where the jump is exponentially close to $\mathbf{1}$. We argue that the only contribution of the order $t^{-1 / 2}$ comes from the circle $\partial D$. Indeed, the circles $\partial C, \partial C_{d}$ give the contribution of the order $t^{-1}$, and the contribution from segments $\Sigma_{E} \cap D$ within the disk $D$ involves the integral of the order

$$
\int_{0}^{\delta}|\mu| \mathrm{e}^{-4 t \mu^{2}} \mathrm{~d} \mu<t^{-1} \int_{0}^{\delta}|\mu| \sqrt{t} \mathrm{e}^{-4 t \mu^{2}} \mathrm{~d}(\mu \sqrt{t})=\mathcal{O}\left(t^{-1}\right)
$$

Henceforth, we have

$$
q_{e r r}^{(1)}(x, t)=2 \mathrm{i} \lim _{k \rightarrow \infty} k\left(E^{(1)}-\mathbf{1}\right)_{12}=\frac{1}{\pi} \int_{\partial D}\left(J_{E^{(1)}}(s)-\mathbf{1}\right)_{12} \mathrm{~d} s+\mathcal{O}\left(t^{-1}\right),
$$

and substituting here

$$
J_{E^{(1)}}=G \cdot F^{\sigma_{3}} \cdot P_{P C} \cdot F^{-\sigma_{3}} G^{-1}, \quad \text { where } \quad F:=\left(\chi(k, \xi) \mathrm{e}^{2 \mathrm{i} t \xi^{2}}(2 t)^{\frac{\mathrm{i} \nu}{2}}\right) \cdot\left(\frac{k-E_{0}}{k-\bar{E}_{0}}\right)^{-n},
$$

and hence

$$
\left(J_{E^{(1)}}-\mathbf{1}\right)_{12}=\left(P_{P C}\right)_{12} F^{2}\left(1+\frac{a_{1}}{k-E_{0}}\right)^{2}-\left(P_{P C}\right)_{21} \frac{\tilde{b}_{1}^{2}}{F^{2}\left(k-\bar{E}_{0}\right)^{2}}+\mathcal{O}\left(t^{-1}\right),
$$

we obtain

$$
q_{e r r}^{(1)}=F\left(k_{0}\right)^{2}\left(1+\frac{a_{1}}{k_{0}-E_{0}}\right)^{2} \frac{1}{\pi} \int_{\partial D} \frac{\mathrm{e}^{\pi \mathrm{i} / 4} \beta_{1} \mathrm{~d} s}{2 \lambda}-\frac{\tilde{b}_{1}^{2}}{F\left(k_{0}\right)^{2}\left(k_{0}-\bar{E}_{0}\right)^{2}} \frac{1}{\pi} \int_{\partial D} \frac{\mathrm{e}^{3 \pi \mathrm{i} / 4} \beta_{2} \mathrm{~d} s}{2 \lambda}+\mathcal{O}\left(t^{-1}\right),
$$

and substituting in the above integrals $\lambda=\sqrt{2 t}\left(s-k_{0}\right)$ and computing the residues, namely

$$
\begin{aligned}
& \frac{1}{\pi} \int_{\partial D} \frac{\mathrm{e}^{\pi \mathrm{i} / 4} \beta_{1} \mathrm{~d} s}{2 \lambda}=\frac{1}{\pi} \int_{\partial D} \frac{\mathrm{e}^{\pi \mathrm{i} / 4} \beta_{1} \mathrm{~d} s}{2 \sqrt{2 t}\left(s-s_{0}\right)}=\frac{\mathrm{e}^{3 \pi \mathrm{i} / 4} \beta_{1}}{\sqrt{2} \sqrt{t}}=\frac{\mathrm{e}^{\pi \mathrm{i} / 4} \sqrt{\pi} 2^{2 \mathrm{i} \nu} \mathrm{e}^{\pi \nu / 2}}{r\left(k_{0}\right) \cdot \Gamma(\mathrm{i} \nu)} \cdot \frac{1}{\sqrt{t}}, \\
& \frac{1}{\pi} \int_{\partial D} \frac{\mathrm{e}^{3 \pi \mathrm{i} / 4} \beta_{2} \mathrm{~d} s}{2 \lambda}=\frac{\mathrm{e}^{5 \pi \mathrm{i} / 4} \beta_{2}}{\sqrt{2} \sqrt{t}}=\frac{\mathrm{e}^{-\pi \mathrm{i} / 4} \sqrt{\pi} 2^{-2 \mathrm{i} \nu} \mathrm{e}^{\pi \nu / 2}}{\overline{r\left(k_{0}\right)} \cdot \Gamma(-\mathrm{i} \nu)} \cdot \frac{1}{\sqrt{t}},
\end{aligned}
$$

we obtain

$$
q_{\text {err }}^{(1)}=\frac{\sqrt{\pi} \mathrm{e}^{\pi \nu / 2}}{B^{2} \sqrt{t}}\left(\frac{\chi^{2}\left(k_{0}\right) \cdot \mathrm{e}^{4 \mathrm{i} t \xi^{2}}(8 t)^{\mathrm{i} \nu}\left(a_{1}-\mathrm{i} B\right)^{2}}{r\left(k_{0}\right) \Gamma(\mathrm{i} \nu) \mathrm{e}^{3 \pi \mathrm{i} / 4}}+\frac{\tilde{b}_{1}^{2} \mathrm{e}^{-\pi \mathrm{i} / 4}(8 t)^{-\mathrm{i} \nu}}{\chi^{2}\left(k_{0}\right) \cdot \mathrm{e}^{4 \mathrm{i} t \xi^{2}} \overline{r\left(k_{0}\right)} \Gamma(-\mathrm{i} \nu)}\right)+\mathcal{O}\left(\frac{\ln t}{t}\right) .
$$


Combining this with (5.24), we obtain

$$
\begin{aligned}
q(x, t) & =\frac{(-1)^{n} 2 B \exp \left[2 \mathrm{i} \varphi(t)-\mathrm{i} \arg \hat{\phi}\left(E_{0}\right)+2 \mathrm{i} \arg \delta\left(E_{0}, \xi\right)\right]}{\cosh \left[2 B(x+4 A t)+\left(2 n+\frac{3}{2}\right) \ln t+\ln \left(\frac{2 \pi}{n ! \Gamma\left(n+\frac{3}{2}\right)} \cdot \frac{\left|\delta\left(E_{0}\right)\right|^{2}\left(16 B^{2}\right)^{2 n+\frac{3}{2}}}{\left|\hat{\phi}\left(E_{0}\right)\right| \sqrt{2 B}}\right)\right]}+ \\
& +\frac{\sqrt{\pi} \mathrm{e}^{\pi \nu / 2}}{B^{2} \sqrt{t}}\left(\frac{\chi^{2}\left(k_{0}\right) \cdot \mathrm{e}^{4 \mathrm{i} t \xi^{2}}(8 t)^{\mathrm{i} \nu}\left(a_{1}-\mathrm{i} B\right)^{2}}{\mathrm{e}^{3 \pi \mathrm{i} / 4} r\left(k_{0}\right) \Gamma(\mathrm{i} \nu)}+\frac{\tilde{b}_{1}^{2} \mathrm{e}^{-\pi \mathrm{i} / 4}}{\chi^{2}\left(k_{0}\right) \cdot \mathrm{e}^{4 \mathrm{i} t \xi^{2}}(8 t)^{\mathrm{i} \nu} \overline{r\left(k_{0}\right)} \Gamma(-\mathrm{i} \nu)}\right)+\mathcal{O}\left(\frac{\ln t}{t}\right) .
\end{aligned}
$$

The latter can be simplified in view of (5.25). Namely, denote the first summand as $q_{s o l}$. Then the second summand can be written as

$$
\frac{\sqrt{\pi} \mathrm{e}^{\pi \nu / 2}}{B^{2} \sqrt{t}}\left[\frac{\chi^{2}\left(k_{0}\right) \mathrm{e}^{4 \mathrm{i} t \xi^{2}}(8 t)^{\mathrm{i} \nu}}{r\left(k_{0}\right) \Gamma(\mathrm{i} \nu)} \cdot \mathrm{e}^{\pi \mathrm{i} / 4}\left(1-\left|\frac{q_{s o l}}{2 B}\right|^{2}\right)+\frac{\mathrm{e}^{3 \pi \mathrm{i} / 4} \cdot \frac{q_{s o l}^{2}}{4 B^{2}}}{\chi^{2}\left(k_{0}\right) \mathrm{e}^{4 i t \xi^{2}(8 t)^{\mathrm{i} \nu}} \overline{r\left(k_{0}\right)} \Gamma(-\mathrm{i} \nu)}\right]
$$

Now we use that

$$
|\Gamma(\mathrm{i} \nu)|^{2}=\frac{\pi}{\nu \sinh (\pi \nu)}, \quad \text { and hence } \quad \frac{\mathrm{e}^{\pi \nu / 2}}{\Gamma(\mathrm{i} \nu)}=\mathrm{e}^{-\mathrm{i} \arg \Gamma(\mathrm{i} \nu)}\left|r\left(k_{0}\right)\right| \sqrt{\frac{\nu}{2 \pi}} .
$$

Denote

$$
\psi:=4 t \xi^{2}+\nu \ln (8 t)+2 \arg \chi\left(k_{0}\right)-\arg r\left(k_{0}\right)-\arg \Gamma(\mathrm{i} \nu)+\frac{\pi}{4},
$$

then (5.28) becomes

$$
\sqrt{\frac{\nu}{2 t}}\left(\mathrm{e}^{\mathrm{i} \psi}\left(1-\left|\frac{q_{\text {sol }}}{2 B}\right|^{2}\right)-\left(\frac{q_{\text {sol }}^{2}}{4 B^{2}}\right) \mathrm{e}^{-\mathrm{i} \psi}\right)
$$

and hence (5.27) becomes

$$
q(x, t)=q_{s o l}+\sqrt{\frac{\nu}{2 t}}\left(\mathrm{e}^{\mathrm{i} \psi}\left(1-\left|\frac{q_{s o l}}{2 B}\right|^{2}\right)-\mathrm{e}^{-\mathrm{i} \psi}\left(\frac{q_{s o l}^{2}}{4 B^{2}}\right)\right)+\mathcal{O}\left(\frac{\ln t}{t}\right),
$$

where

$$
\begin{aligned}
& q_{s o l}=\frac{(-1)^{n} 2 B \exp \left[2 \mathrm{i} \varphi(t)-\mathrm{i} \arg \hat{\phi}\left(E_{0}\right)+2 \mathrm{i} \arg \delta\left(E_{0}, \xi\right)\right]}{\cosh \left[2 B(x+4 A t)+\left(2 n+\frac{3}{2}\right) \ln t+\ln \left(\frac{2 \pi}{n ! \Gamma\left(n+\frac{3}{2}\right)} \cdot \frac{\left|\delta\left(E_{0}\right)\right|^{2}\left(16 B^{2}\right)^{2 n+\frac{3}{2}}}{\left|\hat{\phi}\left(E_{0}\right)\right| \sqrt{2 B}}\right)\right]}, \\
& \psi:=4 t \xi^{2}+\nu \ln (8 t)+2 \arg \chi\left(k_{0}\right)-\arg r\left(k_{0}\right)-\arg \Gamma(\mathrm{i} \nu)+\frac{\pi}{4}
\end{aligned}
$$

\subsubsection{Second refined approximation of $M^{(3)}$}

To deal with those $\gamma$ such that $\{\gamma\} \in\left(\frac{1}{2}, 1\right)$, we introduce another refined approximation of $M^{(3)}$. Namely, by following a similar strategy as in the previous section we now "remove" the $\frac{1}{\zeta}$ term in the $(1,2)$ entry in the asymptotics $(5.7)$ of $L$, and in the $(2,1)$ entry in asymptotics $(5.8)$ of $L_{d}$ at $\infty$,

$$
\left(\begin{array}{cc}
1 & \frac{R_{2}}{\zeta} \\
0 & 1
\end{array}\right) L=\left(\begin{array}{cc}
1+\mathcal{O}\left(\frac{1}{\zeta}\right) & \mathcal{O}\left(\frac{1}{\zeta^{2}}\right) \\
\mathcal{O}\left(\frac{1}{\zeta}\right) & 1+\mathcal{O}\left(\frac{1}{\zeta}\right)
\end{array}\right), \quad\left(\begin{array}{cc}
1 & 0 \\
\frac{R_{2}}{\zeta_{d}} & 1
\end{array}\right) L_{d}=\left(\begin{array}{cc}
1+\mathcal{O}\left(\frac{1}{\zeta_{d}}\right) & \mathcal{O}\left(\frac{1}{\zeta_{d}}\right) \\
\mathcal{O}\left(\frac{1}{\zeta_{d}}\right) & 1+\mathcal{O}\left(\frac{1}{\zeta_{d}}\right)
\end{array}\right)
$$

where

$$
R_{2}=\frac{2 \pi \mathrm{i} n}{n ! \Gamma\left(n+\frac{1}{2}\right)}, n \geq 0, \quad \text { in particular, } R_{2}=0 \quad \text { for } n=0
$$


In keeping with the previous strategy, we introduce

$$
G_{2}=I+\frac{\mathcal{A}_{2}}{k-E_{0}}+\frac{\widetilde{\mathcal{A}}_{2}}{k-\bar{E}_{0}}
$$

and define the refined approximation of $M^{(3)}$ by

$$
M_{\infty}^{(2)}=\left\{\begin{array}{l}
G_{2}\left(\frac{k-E}{k-\bar{E}}\right)^{-n \sigma_{3}}, \quad\left|k-E_{0}\right|>r,\left|k-\bar{E}_{0}\right|>r,\left|k-k_{0}\right|>\delta \\
G_{2} B_{u}\left(\begin{array}{cc}
1 & \frac{R_{2}}{\zeta} \\
0 & 1
\end{array}\right) L(-\phi(k, \xi))^{\sigma_{3} / 2} t^{\gamma \sigma_{3}} \mathrm{e}^{-\mathrm{i} \varphi(t) \sigma_{3}}, \quad\left|k-E_{0}\right|<r \\
G_{2} B_{d}\left(\begin{array}{cc}
1 & 0 \\
\frac{R_{2}}{\zeta_{d}} & 1
\end{array}\right) L_{d}(-\bar{\phi}(\bar{k}, \xi))^{-\sigma_{3} / 2} t^{-\gamma \sigma_{3}} \mathrm{e}^{-\mathrm{i} \varphi(t) \sigma_{3}}, \quad\left|k-\bar{E}_{0}\right|<r \\
G_{2} B_{P C}(k) P_{P C}(\lambda) \cdot\left(\chi(k, \xi) \mathrm{e}^{2 \mathrm{i} t \xi^{2}}(2 t)^{\frac{\mathrm{i} \nu}{2}}\right)^{-\sigma_{3}},\left|k-k_{0}\right|<\delta
\end{array}\right.
$$

with $B_{u}, B_{d}, B_{P C}$ as in (5.13). The error matrix

$$
E^{(2)}:=M^{(3)}\left(M_{\infty}^{(2)}\right)^{-1}
$$

has no discontinuity in the disks $\left|k-E_{0}\right|<r,\left|k-\bar{E}_{0}\right|<r$, and on the circles $\partial C, \partial C_{d}$ the jump is

$$
\begin{aligned}
& J_{E^{(2)}}=G_{2}\left(\begin{array}{cc}
1+\mathrm{O}\left(t^{-1}\right) & \mathrm{O}\left(t^{-2 \gamma+2 n-2}\right) \\
\mathrm{O}\left(t^{2 \gamma-2 n-1}\right) & 1+\mathrm{O}\left(t^{-1}\right)
\end{array}\right) G_{2}^{-1}, k \in \partial C, \\
& J_{E^{(2)}}=G_{2}\left(\begin{array}{cc}
1+\mathrm{O}\left(t^{-1}\right) & \mathrm{O}\left(t^{2 \gamma-2 n-1}\right) \\
\mathrm{O}\left(t^{-2 \gamma+2 n-2}\right) & 1+\mathrm{O}\left(t^{-1}\right)
\end{array}\right) G_{2}^{-1}, k \in \partial C_{d} .
\end{aligned}
$$

We see that estimates in (5.31) are shifted with respect to estimates in (5.20), which allows to use both of them for different ranges of $\gamma$. For $\{\gamma\} \in\left(\frac{1}{2}, 1\right)$ we take

$$
n=\lfloor\gamma\rfloor+1 \text {, }
$$

then $J_{E^{(2)}}$ in $(5.31)$ is of the order

$$
J_{E^{(2)}}=I+\mathcal{O}\left(t^{-1}\right) .
$$

The minimal possible value of $\gamma$ according to (5.2), is $\frac{-1}{4}$, and in this case we take $n=0$. Hence, we are able to handle all the cases $\gamma \geq \frac{-1}{4}$ with constructions in terms of Laguerre polynomials with nonnegative index $n$. The matrix $G_{2}(5.29)$ is determined by the requirement that $M^{(2)}$ is regular at the points $k=E_{0}, k=\bar{E}_{0}$ : similar arguments lead to

$$
\mathcal{A}_{2}=\left(\begin{array}{cc}
0 & b_{2} \\
0 & a_{2}
\end{array}\right), \quad \widetilde{\mathcal{A}}_{2}=\left(\begin{array}{cc}
\widetilde{a}_{2} & 0 \\
\widetilde{b}_{2} & 0
\end{array}\right)
$$

where $a_{2}, b_{2}, \widetilde{a}_{2}, \widetilde{b}_{2}$ satisfy the system (5.21) with $H, H_{d}$ replaced by $H_{2}, H_{2, d}$, respectively, and $a, b, \widetilde{a}, \widetilde{b}$ replaced with $a_{2}, b_{2}, \widetilde{a}_{2}, \widetilde{b}_{2}$, respectively. Hence, $a_{2}, b_{2}, \widetilde{a}_{2}, \widetilde{b}_{2}$ are defined by (5.22), (5.23), where we replace $H, H_{d}$ with $H_{2}, H_{2, d}$. Here

$$
\begin{aligned}
& H_{2}=R_{2} t^{2 n-2 \gamma-1} \lim _{k \rightarrow E_{0}}\left(\frac{k-E_{0}}{\left(k-\bar{E}_{0}\right) z}\right)^{-2 n}(-\phi)^{-1} \mathrm{e}^{2 \mathrm{i} \varphi(t)}=\frac{R_{2}(-1)^{n+1} t^{2 n-2 \gamma-1}\left(2 B z^{\prime}\right)^{2 n+\frac{1}{2}} \mathrm{e}^{2 \mathrm{i} \varphi(t)}}{\hat{\phi}\left(E_{0}\right) \delta\left(E_{0}, \xi\right)^{-2} \sqrt{2 B}}, \\
& H_{2, d}=R_{2} t^{2 n-2 \gamma-1} \lim _{k \rightarrow \bar{E}_{0}}\left(\frac{z_{d}\left(k-E_{0}\right)}{k-\bar{E}_{0}}\right)^{2 n}\left(-\bar{\phi}_{d}(\bar{k})\right)^{-1} \mathrm{e}^{-2 \mathrm{i} \varphi(t)}=\frac{R_{2}(-1)^{n+1} t^{2 n-2 \gamma-1}\left(2 B z_{d}^{\prime}\right)^{2 n+\frac{1}{2}}}{\hat{\hat{\phi}\left(E_{0}\right)}\left[\overline{\delta\left(E_{0}, \xi\right)}\right]^{-2} \sqrt{2 B} \mathrm{e}^{2 \mathrm{i} \varphi(t)}},
\end{aligned}
$$




$$
\bar{H}_{2, d}=-H_{2} \text {. }
$$

Let us observe for future reference that $\frac{H_{2}}{2 B z^{\prime}}$ equals $\frac{2 B z^{\prime}}{H}$, if we change in the latter $n \rightarrow n-1$. As a consequence, we have that $b_{2}$ coincides with $\tilde{b}_{1}$ and $\tilde{a}_{2}+\mathrm{i} B$ coincides with $a_{1}-\mathrm{i} B$, if we change in the latter $n \rightarrow n-1$. Coming back to the computation of $q_{\infty}^{(2)}$, we see that again $G_{2}$ does not contribute into asymptotics (5.31), and

$$
\begin{aligned}
q_{\infty}^{(2)}= & 2 i \lim _{k \rightarrow \infty} k M_{\infty, 12}^{(2)}=-2 i \lim _{k \rightarrow \infty} \overline{k M_{\infty, 21}^{(2)}}=2 i b_{2}=-2 i \overline{\widetilde{b}_{2}}=\frac{-4 \mathrm{i} B \cdot \frac{H_{2}}{\left|H_{2}\right|} \cdot \frac{\bar{z}^{\prime}}{\left|z^{\prime}\right|}}{\frac{2 B\left|z^{\prime}\right|}{|H|}+\frac{|H|}{2 B\left|z^{\prime}\right|}} \\
& =\frac{2 B(-1)^{n-1} \mathrm{e}^{2 \mathrm{i} \varphi(t)} \mathrm{e}^{-\mathrm{i} \arg \hat{\phi}\left(E_{0}\right)} \mathrm{e}^{2 \mathrm{i} \arg \delta\left(E_{0}, \xi\right)} \mathrm{e}^{\mathrm{i}\left(2 n-\frac{1}{2}\right) \arg \left(z^{\prime}\right)}}{\cosh \left[2 B(x+4 A t)+\left(2 n-\frac{1}{2}\right) \ln t+\ln \frac{2 \pi n\left|\delta\left(E_{0}, \xi\right)\right|^{2}}{n ! \Gamma\left(n+\frac{1}{2}\right)\left|\hat{\phi}\left(E_{0}\right)\right| \sqrt{2 B}}+\left(2 n-\frac{1}{2}\right) \ln \left(2 B\left|8 B+\frac{2 \mathrm{i} \rho \ln t}{B t}\right|\right)\right]} \\
& =\frac{2 B(-1)^{n-1} \mathrm{e}^{2 \mathrm{i} \varphi(t)} \mathrm{e}^{-\mathrm{i} \arg \hat{\phi}\left(E_{0}\right)} \mathrm{e}^{2 \mathrm{i} \arg \delta\left(E_{0}, \xi\right)}}{\cosh \left[2 B(x+4 A t)+\left(2 n-\frac{1}{2}\right) \ln t+\ln \frac{2 \pi n\left(16 B^{2}\right)^{2 n-\frac{1}{2}}\left|\delta\left(E_{0}, \xi\right)\right|^{2}}{n ! \Gamma\left(n+\frac{1}{2}\right)\left|\hat{\phi}\left(E_{0}\right)\right| \sqrt{2 B}}\right]}+\mathcal{O}\left(\frac{\ln t}{t}\right)
\end{aligned}
$$

for $n \geq 1$, and $q_{\infty}^{(2)}=0$ for $n=0$. We see that (5.32) coincides with (5.24), if we replace in the latest $n$ with $n-1$. Furthermore,

$$
q(x, t)=q_{\infty}^{(2)}+q_{\text {err }}^{(2)}, \quad \text { where } \quad q_{\text {err }}^{(2)}:=2 \mathrm{i} \lim _{k \rightarrow \infty} k\left(E^{(2)}\right)_{12}
$$

and it follows from $J_{E^{(2)}}=I+\mathcal{O}\left(t^{-1 / 2}\right)$ that

$$
q_{\text {err }}^{(2)}=\mathcal{O}\left(t^{-1 / 2}\right)
$$

Computing $q_{e r r}^{(2)}$ in the same way as in Subsection 5.2.2, we find

$$
\begin{aligned}
& q_{e r r}^{(2)}(x, t)=2 \mathrm{i} \lim _{k \rightarrow \infty} k\left(E^{(2)}-\mathbf{1}\right)_{12}=\frac{1}{\pi} \int_{\partial D}\left(J_{E^{(2)}}(s)-\mathbf{1}\right)_{12} \mathrm{~d} s+\mathcal{O}\left(t^{-1}\right)= \\
& \quad=\frac{\sqrt{\pi} \mathrm{e}^{\pi \nu / 2}}{B^{2} \sqrt{t}}\left(\frac{\mathrm{e}^{-3 \pi \mathrm{i} / 4} \chi^{2}\left(k_{0}\right) \mathrm{e}^{4 \mathrm{i} t \xi^{2}}(8 t)^{\mathrm{i} \nu}\left(\tilde{a}_{2}+\mathrm{i} B\right)^{2}}{r_{0} \cdot \Gamma(\mathrm{i} \nu)}+\frac{\mathrm{e}^{-\pi \mathrm{i} / 4} b_{2}^{2}}{\chi^{2}\left(k_{0}\right) \mathrm{e}^{4 \mathrm{i} t \xi^{2}}(8 t)^{\mathrm{i} \nu} \overline{r_{0}} \Gamma(-\mathrm{i} \nu)}\right)+\mathcal{O}\left(\frac{\ln t}{t}\right) .
\end{aligned}
$$

and due to the previous observation the latter equals (5.27), where we substitute $n$ with $n-1$. Let us notice, that all the estimates in subsections 5.2.1, 5.2.3 are uniform with respect to finite shifts of the parameter $\rho$. We thus obtain the statements of Theorems 1, 2 .

\section{References}

[1] M.J. Ablowitz and H. Segur, Solitons and the Inverse Scattering Transform (SIAM, Philadelphia, 1981).

[2] E. D. Belokolos, A. I. Bobenko, V. Z. Enol'skii, A. R. Its, and V. B. Matveev, Algebrogeometric approach to nonlinear integrable equations. Springer Series in Nonlinear Dynamics, Springer-Verlag, Berlin (1994).

[3] Bertola, M., Minakov, A. Laguerre polynomials and transitional asymptotics of the modified Korteweg-de Vries equation for step-like initial data. Anal.Math.Phys. (2018). https://doi.org/10.1007/s13324-018-0273-1 
[4] R.F.Bikbaev, Complex Whitham deformations in the problems with "integrable instability", Theor. Math. Phys.,104, no.3 (1995), 1078-1097.

[5] R.F.Bikbaev, Saturation of modulational instability via complex Whitham deformations: Schrödinger equation, J. Math. Sciences 85 (1997), no.1, 1596-1604.

[6] Gino Biondini and Dionyssios Mantzavinos, Long-Time Asymptotics for the Focusing Nonlinear Schrödinger Equation with Nonzero Boundary Conditions at Infinity and Asymptotic Stage of Modulational Instability, Communications on Pure and Applied Mathematics Volume70, Issue12 (2017), 2300-2365

[7] A. Boutet de Monvel and V.P. Kotlyarov, The focusing nonlinear Schrödinger equation on the quarter plane with time-periodic boundary condition: a Riemann-Hilbert approach, J. Inst. Math. Jussieu 6 (2007), no. 4, 579-611.

[8] A. Boutet de Monvel, A.R. Its, and V.P. Kotlyarov, Long-time asymptotics for the focusing NLS equation with time-periodic boundary condition on the half-line, Comm. Math. Phys. 290 (2009), no. 2, 479-522.

[9] A. Boutet de Monvel, V.P. Kotlyarov, and D. Shepelsky, Decaying long-time asymptotics for the focusing NLS equation with periodic boundary condition, Int. Math. Res. Notices 2009, no. $3,547-577$.

[10] A. Boutet de Monvel, V.P. Kotlyarov, and D. Shepelsky, Focusing NLS equation: Long-time Dynamics of the Step-like Initial Data// International Mathematics Research Notices, Vol. 2011 (2011), no.7, 1613-1653.

[11] R. Buckingham and S. Venakides, Long-time asymptotics of the nonlinear Schrödinger equation shock problem, Comm. Pure Appl. Math. 60 (2007), no. 9, 1349-1414.

[12] P.Deift and X.Zhou A steepest descent method for oscillatory Riemann-Hilbert problems, Bull. Amer. Math. Soc. (N.S.) 26 (1992), no. 1, 119-123.

[13] P.Deift, A.Its, and X.Zhou, Long-time asymptotics for integrable nonlinear wave equations, in: Important developments in soliton theory, Springer Ser. Nonlinear Dynam., 1993, 181-204.

[14] P.Deift and X.Zhou, A steepest descent method for oscillatory Riemann-Hilbert problems. Asymptotics for the MKdV equation, Ann. of Math. 137 (1993) no. 2, 295-368.

[15] P. Deift, S. Venakides, and X. Zhou, The collisionless shock region for the long-time behavior of solutions of the KdV equation, Commun. Pure Appl. Math., 47 (1994), 199-206.

[16] P.A. Deift, A.R. Its, and X. Zhou, A Riemann-Hilbert approach to asymptotic problems arising in the theory of random matrix models, and also in the theory of integrable statistical mechanics, Ann. of Math., 146 (1997), 149-235.

[17] P. Deift, S. Venakides, and X. Zhou, New results in small dispersion KdV by an extension of the steepest-descent method for Riemann-Hilbert problems, Int. Math. Res. Notices, 6 (1997), $285-299$.

[18] P. Deift, T. Kricherbauer, K. T.-R. McLaughlin, S. Venakides, and X. Zhou, Uniform asymptotics for polynomials orthogonal with respect to varying exponential weights and applications to universality questions in random matrix theory, Commun. Pure Appl. Math., 52 (1999), $1335-1425$. 
[19] Deift P. Orthogonal polynomials and random matrices: a Riemann - Hilbert approach / P. Deift. - NY.: CIMS, 1999.

[20] P Dubard and V B Matveev, Multi-rogue waves solutions to the focusing NLS equation and the KP-I equation Natural Hazards and Earth System Sciences 11 (3), (2011), 667-672

[21] P Dubard and V B Matveev, Multi-rogue waves solutions: from the NLS to the KP-I equation, Nonlinearity, 26 (2013), R93-R125;

[22] I. Egorova, Z. Gladka, V. P. Kotlyarov, and G. Teschl, Long-time asymptotics for the Kortewegde Vries equation with step-like initial data. Nonlinearity 26 (2013), 1839-1864.

[23] G.A. El and M.A.Hoefer, Dispersive shock waves and modulation theory, Physica D: Nonlinear Phenomena, 333, (2016) 11-65. doi:10.1016/j.physd.2016.04.006

[24] L. D. Faddeev and L. A. Takhtajan, Hamiltonian methods in the theory of solitons. Springer Series in Soviet Mathematics. Springer-Verlag, Berlin (1987).

[25] A. V. Gurevich and L. P. Pitaevskii, Zh. Eksp. Teor. Fiz., 65 (1973), 590-604.

[26] A. R. Its, Asymptotic behavior of the solutions to the nonlinear Schrödinger equation, and isomonodromic deformations of systems of linear differential equations, Soviet. Math. Dokl. 24 (1981), 452-456.

[27] M Kamalian, A Vasylchenkova, D Shepelsky, JE Prilepsky, SK Turitsyn, Signal Modulation and Processing in Nonlinear Fibre Channels by Employing the Riemann-Hilbert Problem, Journal of Lightwave Technology 36 (24), (2018), 5714-5727

[28] E.Ya. Khruslov, Asymptotic behavior of the solution of the Cauchy problem for the Kortewegde Vries equation with steplike initial data, Math. USSR-Sb. 28 (1976), no. 2, 229-248.

[29] V.P. Kotlyarov and E.Ya. Khruslov, Solitons of the nonlinear Schrödinger equation, which are generated by the continuous spectrum, Teoret. Mat. Fiz. 68 (1986), no. 2, 172-186.

[30] V.P. Kotlyarov, Influence of double continuous spectrum of the Dirac operator on the nonlinear Schrödinger equation asymptotic solitons. Matem. zametki, v.49, N 2, 1991, pp.84-94; Math. Notes, v.49, N 1-2, 1991, pp.172-180.

[31] V. P. Kotlyarov and A. Minakov, Riemann-Hilbert problem to the modified Korteveg-de Vries equation: Long-time dynamics of the steplike initial data. J. Math. Phys. 51 (2010), 093506.

[32] V. P. Kotlyarov and A. A. Minakov Step-initial function to the mkdv equation: hyper-elliptic long-time asymptotics of the solution. Journal of mathematical physics, analysis, geometry Vol. 8, 1, (2012), 38-62.

[33] V. Kotlyarov, and D. Shepelsky, Planar unimodular Baker-Akhiezer functionfor the nonlinear Schrödinger equation// Annals of Mathematical Sciences and Applications, 2017, Volume 2, Number 2, 343-384

[34] G. Litvinchuk and I. Spitkovskii, Factorization of measurable matrix functions / — Basel: Birkhauser Verlag, 1987. - $51 \mathrm{p}$.

[35] V B Matveev, A O Smirnov, Solutions of the Ablowitz-Kaup-Newell-Segur hierarchy equations of the rogue wave type: A unified approach, Theoretical and Mathematical Physics 186 (2), (2016), 156-182 
[36] V B Matveev, A O Smirnov, AKNS and NLS hierarchies, MRW solutions, Pn breathers, and beyond, Journal of Mathematical Physics 59 (9), (2018), 091419

[37] V.Yu.Novokshenov Temporal asymptotics for soliton equations in problems with step initial conditions. J. Math. Sciences, 125 (2005), no.5, 717-747.

[38] A Vasylchenkova, JE Prilepsky, D Shepelsky, A Chattopadhyay, Direct nonlinear Fourier transform algorithms for the computation of solitonic spectra in focusing nonlinear Schrǒdinger equation, Communications in Nonlinear Science and Numerical Simulation 68, (2019), 347371.

[39] V.E. Zakharov and A.B. Shabat, An exact theory of two-dimensional self-focusing and onedimensional automodulation of waves in a nonlinear medium, Soviet Phys. JETP 34 (1972), $62-78$.

[40] Zhou X. The Riemann-Hilbert problem and inverse scattering // SIAM J. Math. Anal. 1989. - Vol. 20. - P. 966-986. 